Tohoku Math. J.

54 (2002), 195-226

\title{
CLASSIFICATION OF DEGENERATIONS OF CURVES OF GENUS THREE VIA MATSUMOTO-MONTESINOS' THEOREM
}

Dedicated to Professor Tadao Oda on his sixtieth birthday

TADASHI ASHIKAGA AND MizuHo IsHIZAKA

(Received May 26, 2000, revised December 27, 2000)

Abstract. We classify singular fibers of curves of genus three and determine their topological monodromies and the strata of their moduli points.

Introduction. Let $\phi: S \rightarrow \Delta$ be a proper surjective holomorphic map from a complex surface $S$ to the unit disk $\Delta=\{t \in C|| t \mid<1\}$ such that $\phi^{-1}(t)$ is a smooth curve of genus $g \geq 1$ for any $t \in \Delta^{*}=\Delta-\{0\}$. We call $\phi$ a degeneration of curves of genus $g$ and call $F=\phi^{-1}(0)$ the singular fiber. The most fundamental problem of degenerations of curves is to classify singular fibers, their monodromies and moduli points. After the monumental work of Kodaira [Ko] for elliptic curves, Namikawa-Ueno [NU1], [NU2] classified singular fibers of genus two and determined their homological monodromies and the limits of their period matrices.

The main result of this paper (Theorem 4.3) is to classify singular fibers of genus three and determine their topological monodromies and the strata of their moduli points. More precisely, we explicitly determine the conjugacy class, arising from the usual monodromy action for each degeneration, of the mapping class group of a Riemann surface of genus three and determine the topological type of the stable curve which is the limit of the moduli map in Deligne-Mumford's compactified moduli space $\overline{\boldsymbol{M}}_{3}$ of genus three.

Our basic tools are the following Theorems (A) and (B), which was first studied by Nielsen [Ni2], developed by [Cl], [Im], [ES], [ST], [AMO], etc., and finally settled by Matsumoto-Montesinos [MM1], [MM2]:

(A) ([MM2, Theorem 1]) The conjugacy class, realized as the topological monodromy of a degeneration of curves, of the mapping class group of a Riemann surface of genus $g \geq 2$ is represented by a pseudo-periodic map of negative type. Conversely, any conjugacy class of pseudo-periodic map of negative type is realized as the topological monodromy of a certain degeneration of curves.

(B) ([MM2, Theorem 2]) The conjugacy class of a pseudo-periodic map $f: \Sigma_{g} \rightarrow \Sigma_{g}$ of negative type of a smooth curve $\Sigma_{g}$ of genus $g$ is determined by the following data: An admissible system of cut curves $\mathcal{C}=\amalg C_{i}$ on $\Sigma_{g}$, the action of $f$ on the oriented graph $G_{\mathcal{C}}$ induced by $\mathcal{C}$, the screw numbers of $f$ around each annulus of $C_{i}$ and the valency data of

2000 Mathematics Subject Classification. Primary 14D06; Secondary 14H45, 14H15, 57M99, 30F99. 
the periodic maps which stabilize the connected components of $\Sigma_{g}-\mathcal{C}$. It is equivalent to determining the action of $f$ on $G_{\mathcal{C}}$ and Matsumoto-Montesinos' generalized quotient $\Sigma_{g} \rightarrow$ $\mathcal{S}_{f}$ of $f$.

Our strategy for the classification is divided into the following four steps:

(1) We classify admissible systems $\mathcal{C}$ of cut curves on $\Sigma_{3}$ (Lemma 3.2). This is equivalent to classifying the stable curves of genus three, which is well-known.

(2) We classify cyclic automorphisms of the graph $G_{\mathcal{C}}$ (Lemma 3.4). This is equivalent to classifying the cyclic automorphisms of the dual graphs of stable curves of genus three.

(3) We classify periodic homeomorphisms modulo isotopy of a Riemann surface with boundary, which is realized as a connected component of $\Sigma_{3}-\mathcal{C}$. This is equivalent to classifying the cyclic automorphisms of an irreducible $n$-pointed stable curve of genus $\leq 3$ and small $n$ admitting permutations of marked points. For this purpose, we first classify the valency data of the cyclic automorphisms of a closed Riemann surface of genus $\leq 3$ by using Nielsen and Harvey's formula (Lemma 1.4). The sufficiency of these conditions is proved in $\$ 4.4$. We apply the result to Riemann surfaces with boundary case by case in $\$ 2.2$.

(4) For each action on the graph $G_{\mathcal{C}}$ classified in (2), we choose periodic homeomorphisms of "parts" of a Riemann surface described in (3) compatible to this acion. In this way, we classfiy all conjugacy classes of pseudo-periodic maps of negative type of genus three (Proposition 3.8).

In Section 1 through Section 3, we avoid the language of algebraic geometry and discuss in terms of the mapping class group of a Riemann surface. In Section 4, we translate this result into the algebro-geometric statement on degenerations. In summary, the degeneration $\phi: S \rightarrow \Delta$ which we obtain has the following properties:

(a) The stable curve which is the moduli point of $\phi$ has the dual graph $G_{\mathcal{C}}$.

(b) The topological monodromy of $\phi$ is determined by the data which we have at the starting point for characterizing the pseudo-periodic map $f$ of negative type.

(c) The topological type of the singular fiber $\phi^{-1}(0)$ coincides with $S_{f}$. We obtain $S_{f}$ by substitution in the sense of Subsection 3.7 of the corresponding "partial" generalized quotient space in Table 1 to the graph in Table 2 or Table 3.

For a numerical classification of singular fibers of genus three, see Uematsu [Ue]. For an algebro-geometric study of a pencil of genus three, see Mendes-Lopes [Me]. The algebrogeometric meaning of Matsumoto-Montesinos' theory is partially studied by Takamura [Ta] and Terasoma $[\mathrm{Te}]$.

The authors express their special thanks to Professor Yukio Matsumoto for explaining to us the works of Matsumoto-Montesinos [MM1], [MM2] and giving us useful advice. They also thank Professor Kazuhiro Konno, Professor Toshiyuki Akita, Professor Shigeru Takamura, Professor Makoto Matsumoto and Professor Takeshi Kajiwara for discussions. They thank the referee for pointing out mistakes and giving them useful advice. They thank Professor Tadao Oda for constant encouragement. 
1. Periodic map. In this section, we first review a part of Nielsen's work [N1] and then apply it to classify conjugacy classes of periodic homeomorphisms of closed surfaces of genus $\leq 3$.

1.1. By a surface $\Sigma$, we mean an oriented connected real 2-dimensional manifold with or without boundary. When we emphasize its complex structure, we call $\Sigma$ a Riemann surface. Let $f: \Sigma \rightarrow \Sigma$ be a periodic orientation-preserving homeomorphism (or periodic map, for short) of order $n \geq 2$. Let $P$ be a point on $\Sigma$. There is a positive integer $\alpha(P)$ such that the points $P, f(P), \ldots, f^{\alpha(P)-1}(P)$ are mutually distinct and $f^{\alpha(P)}(P)=P$. If $\alpha(P)=n$, we call the point $P$ a simple point of $f$, while if $\alpha(P)<n$, we call $P$ a multiple point of $f$. Note that a multiple point is an isolated and interior point of $\Sigma$.

Let $C$ be an oriented simple closed curve on $\Sigma$, which we sometimes write $\vec{C}$ to emphasize its orientation. Let $m=m(\vec{C})$ be the smallest positive integer such that $f^{m}(\vec{C})=$ $\vec{C}$, i.e., $f^{m}(C)=C$ as a set and $f^{m}$ preserving the orientation of $C$. The restriction of $f^{m}$ to $\vec{C}$ is a periodic map of order, say, $\lambda \geq 1$. Note that $n=m \lambda$. Let $Q$ be any point on $C$, and suppose that the images of $Q$ under the iteration of $f^{m}$ are ordered as $\left(Q, f^{m \sigma}(Q), f^{2 m \sigma}(Q), \ldots, f^{(\lambda-1) m \sigma}(Q)\right)$ viewed in the direction of $\vec{C}$, where $\sigma$ is an integer with $0 \leq \sigma \leq \lambda-1$ and $\operatorname{gcd}(\sigma, \lambda)=1$. Let $\delta$ be the integer which satisfies

$$
\sigma \delta \equiv 1(\bmod \lambda), \quad 0 \leq \delta \leq \lambda-1 .
$$

Then the action of $f^{m}$ on $\vec{C}$ is the rotation of angle $2 \pi \delta / \lambda$ with a suitable parametrization of $\vec{C}$ as an oriented circle. Nielsen called the triple $(m, \lambda, \sigma)$ the valency of $\vec{C}$ with respect to $f$.

He also defined the valency of a boundary curve (i.e., a connected component of the boundary $\partial \Sigma$ ) as its valency with respect to $f$ assuming it has the orientation induced by the surface $\Sigma$. The valency of a multiple point $P$ is defined to be the valency of the boundary curve $\partial D_{P}$, oriented from the outside of a disk neighborhood $D_{P}$ of $P$.

Let $\Pi: \Sigma \rightarrow \Sigma^{\prime}$ be the $n$-fold cyclic covering associated with $f$, where $\Sigma^{\prime}$ is the quotient surface of $\Sigma$ with respect to $f$. The multiple points of $f$ coincide with the ramification points of $\Pi$. We define the valency of a branch point $P$ on $\Sigma^{\prime}$ as the valency of a ramification point on $\Pi^{-1}(P)$.

By Nielsen's theorem [N1, §11], the conjugacy class of periodic maps is completely determined by the set of valencies of the multiple points and boundary curves.

A periodic map of a bounded surface is easily extended to that of a closed surface by the following:

LEMMA 1.2. Let $\Sigma$ be an oriented surface whose boundary is a disjoint union of simple closed curves $\partial_{1}, \ldots, \partial_{k}$, and let $f: \Sigma \rightarrow \Sigma$ be a periodic map. Let $\left(m_{i}, \lambda_{i}, \sigma_{i}\right)$ $(1 \leq i \leq k)$ be the valency of $\partial_{i}$ with respect to $f$. Then there exists a closed surface $\tilde{\Sigma}$ which contains $\Sigma$, and a periodic map $\tilde{f}: \tilde{\Sigma} \rightarrow \tilde{\Sigma}$ such that

(i) the complement $\tilde{\Sigma}-\Sigma$ is a disjoint union of $k$ open disks $\bigsqcup_{1 \leq i \leq k} D_{i}$,

(ii) the restriction $\left.\tilde{f}\right|_{\Sigma}$ coincides with $f$,

(iii) the multiple points of $\tilde{f}$ on $\tilde{\Sigma}-\Sigma$ are the centers $P_{i}$ of the disks $D_{i}(1 \leq i \leq k)$ and the valency at $P_{i}$ with respect to $\tilde{f}$ coincides with the valency $\left(m_{i}, \lambda_{i}, \sigma\right)$. 
PROOF. For each $\partial_{i}(1 \leq i \leq k)$, we have a parametrization $\theta_{i}$ with $\partial_{i} \simeq S^{1}=$ $\left\{\exp \left(2 \pi \sqrt{-1} \theta_{i}\right) \mid 0 \leq \theta_{i}<1\right\}$ such that $\left.f^{m}\right|_{\partial_{i}}: \partial_{i} \rightarrow \partial_{i}$ is given by $\theta_{i} \mapsto \theta_{i}+\delta_{i} / \lambda_{i}$ $(\bmod \boldsymbol{Z})$, where $\delta_{i}$ is the integer determined by $\left(m_{i}, \lambda_{i}, \sigma_{i}\right)$ as in (1.1.1). The curve $\partial_{i}$ is mapped by $f$ to one of the boundary curves, which we write $\partial_{f(i)}(1 \leq f(i) \leq k)$. Under the above parametrization, the map $\left.f\right|_{\partial_{i}}: \partial_{i} \rightarrow \partial_{f(i)}$ is written as $\theta_{i} \mapsto \theta_{f(i)}=F_{i}\left(\theta_{i}\right)$, where $F_{i}$ is a continuous function of $\theta_{i}$. Now let $\tilde{\Sigma}$ be a closed oriented surface obtained from $\Sigma$ and $k$ closed disks $D_{i}(1 \leq i \leq k)$ by identifying $\partial_{i}$ and the boundary $\partial D_{i}$. We define the parametrization $D_{i}=\left\{r_{i} \exp \left(2 \pi \sqrt{-1} \theta_{i}\right) \mid 0 \leq r_{i} \leq 1,0 \leq \theta_{i}<1\right\}$ which is compatible with the previous parametrization of $\partial_{i}$ at $\partial D_{i}$. We define the map $\tilde{f}: \tilde{\Sigma} \rightarrow \tilde{\Sigma}$ by $\tilde{f}=f$ on $\Sigma \subset \tilde{\Sigma}$, and by $r_{i} \exp \left(2 \pi \sqrt{-1} \theta_{i}\right) \mapsto r_{i} \exp \left(2 \pi \sqrt{-1} F_{i}\left(\theta_{i}\right)\right)$ on $D_{i}(1 \leq i \leq k)$. Then $\tilde{f}$ satisfies the required properties.

Q.E.D.

1.3. Let $\Sigma_{g}$ be a closed surface of genus $g \geqq 2$, and let $\Gamma_{g}$ be its mapping class group. In the isotopy class of a periodic map of $\Sigma_{g}$, one can choose a representative which is an analytic automorphism under a certain complex structure on $\Sigma_{g}$ ([Ni1], [B2, Theorem 1] or in more generalized form [Ke])

Now let $f: \Sigma_{g} \rightarrow \Sigma_{g}$ be a cyclic analytic automorphism of order $n$, and let $\Pi: \Sigma \rightarrow$ $\Sigma^{\prime}$ be the corresponding $n$-fold cyclic covering. Let $g^{\prime}$ be the genus of $\Sigma^{\prime}$. We denote by $\lambda_{1}, \ldots, \lambda_{l}$ the ramification indices of $\Pi$ and let $\left(n / \lambda_{i}, \lambda_{i}, \sigma_{i}\right)(1 \leq i \leq l)$ be the valencies of the branch points. The following are known:

(i) (the Hurwitz formula) $2(g-1) / n=2\left(g^{\prime}-1\right)+\sum_{i=1}^{l}\left(1-1 / \lambda_{i}\right)$.

(ii) (Nielsen $[\mathrm{Ni} 1,(4.6)]) \quad \sum_{i=1}^{l} \sigma_{i} / \lambda_{i}$ is an integer.

(iii) (Wiman [W]) $n \leq 4 g+2$.

(iv) (Harvey $[\mathrm{H}]$ ) Assume $g \geq 2$. Set $M=\operatorname{lcm}\left(\lambda_{1}, \ldots, \lambda_{l}\right)$. Then we have:

(1) $\operatorname{lcm}\left(\lambda_{1}, \ldots, \widehat{\lambda_{i}}, \ldots, \lambda_{l}\right)=M$ for all $i$, where $\widehat{\lambda_{i}}$ denotes the omission of $\lambda_{i}$.

(2) $M$ divides $n$, and if $g^{\prime}=0$, then $M=n$.

(3) $l \neq 1$, and, if $g^{\prime}=0$, then $l \geq 3$.

(4) If $2 \mid M$, the number of $\lambda_{1}, \ldots, \lambda_{l}$ which are divisible by the maximal power of 2 dividing $M$ is even.

Conversely, there exists a cyclic analytic automorphism of $\Sigma_{g}$ whose covering degree, ramification indices and the genus of the base surface satisfy the above conditions (i) and (iv), (1)-(4).

Here is a comment on the condition (ii). Let $\Sigma_{g}^{\circ}$ be a surface obtained from $\Sigma$ by removing all the open disks around the multiple points of $f$. Then we obtain the condition (ii) by applying the formula $[\mathrm{Ni} 1,(4.6)]$ to the restriction $\left.f\right|_{\Sigma_{g}^{\circ}}$.

Now we classify the conjugacy classes of periodic maps of closed surfaces with $1 \leq$ $g \leq 3$. By Nielsen's theorem [Ni1, $\$ 11]$, it suffices to classify the order of the map and the valencies of multiple points. For brevity's sake, if we have the data of valencies $\left(n / \lambda_{i}, \lambda_{i}, \sigma_{i}\right)$ $(1 \leq i \leq l)$, we symbolically write $\sigma_{1} / \lambda_{1}+\cdots+\sigma_{l} / \lambda_{l}$ which we will call the total valency. We also write the order $n$ of the map and the genus $g^{\prime}$ of $\Sigma^{\prime}$. However if $g^{\prime}=0$, the genus is omitted. Then: 
LEMMA 1.4. Non-identical conjugacy classes of periodic maps of closed surfaces of genus $1 \leq g \leq 3$ are classified as follows:

(i) $g=1$.

(1) $n=6 ; 1 / 6+1 / 3+1 / 2,5 / 6+2 / 3+1 / 2$.

(2) $n=4 ; 1 / 4+1 / 4+1 / 2,3 / 4+3 / 4+1 / 2$.

(3) $n=3 ; 1 / 3+1 / 3+1 / 3,2 / 3+2 / 3+2 / 3$.

(4) $n=2 ; 1 / 2+1 / 2+1 / 2+1 / 2$.

(5) $g^{\prime}=1, n$ is arbitrary and $\Pi: \Sigma \rightarrow \Sigma^{\prime}$ is an unramified covering.

(ii) $g=2$.

(1) $n=10 ; 1 / 10+2 / 5+1 / 2,3 / 10+1 / 5+1 / 2,7 / 10+4 / 5+1 / 2,9 / 10+3 / 5+1 / 2$.

(2) $n=8 ; 1 / 8+3 / 8+1 / 2,5 / 8+7 / 8+1 / 2$.

(3) $n=6 ; 1 / 6+1 / 6+2 / 3,5 / 6+5 / 6+1 / 3,1 / 3+2 / 3+1 / 2+1 / 2$.

(4) $n=5 ; 1 / 5+1 / 5+3 / 5,1 / 5+2 / 5+2 / 5,2 / 5+4 / 5+4 / 5,3 / 5+3 / 5+4 / 5$.

(5) $n=4 ; 1 / 4+3 / 4+1 / 2+1 / 2$.

(6) $n=3 ; 1 / 3+1 / 3+2 / 3+2 / 3$.

(7) $n=2 ; 1 / 2+1 / 2+1 / 2+1 / 2+1 / 2+1 / 2$.

(8) $g^{\prime}=1, n=2$ and $1 / 2+1 / 2$.

(iii) $g=3$.

(1) $n=14 ; 11 / 14+5 / 7+1 / 2,3 / 14+2 / 7+1 / 2,13 / 14+4 / 7+1 / 2$, $1 / 14+3 / 7+1 / 2,9 / 14+6 / 7+1 / 2,5 / 14+1 / 7+1 / 2$.

(2) $n=12 ; 11 / 12+7 / 12+1 / 2,1 / 12+5 / 12+1 / 2,11 / 12+3 / 4+1 / 3$, $1 / 12+1 / 4+2 / 3,7 / 12+3 / 4+2 / 3,5 / 12+1 / 4+1 / 3$.

(3) $n=9 ; 8 / 9+4 / 9+2 / 3,1 / 9+5 / 9+1 / 3,7 / 9+5 / 9+2 / 3$, $2 / 9+4 / 9+1 / 3,8 / 9+7 / 9+1 / 3,1 / 9+2 / 9+2 / 3$.

(4) $n=8 ; 7 / 8+3 / 8+3 / 4,1 / 8+5 / 8+1 / 4,5 / 8+5 / 8+3 / 4$, $3 / 8+3 / 8+1 / 4,7 / 8+7 / 8+1 / 4,1 / 8+1 / 8+3 / 4$

(5) $n=7 ; 6 / 7+6 / 7+2 / 7,1 / 7+1 / 7+5 / 7,6 / 7+5 / 7+3 / 7$, $1 / 7+2 / 7+4 / 7,6 / 7+4 / 7+4 / 7,1 / 7+3 / 7+3 / 7$ $5 / 7+5 / 7+4 / 7,2 / 7+2 / 7+3 / 7$.

(6) $n=6 ; 5 / 6+1 / 6+1 / 2+1 / 2,5 / 6+1 / 3+1 / 3+1 / 2,1 / 6+2 / 3+2 / 3+1 / 2$.

(7) $n=4 ; 3 / 4+3 / 4+3 / 4+3 / 4,1 / 4+1 / 4+1 / 4+1 / 4$, $3 / 4+3 / 4+1 / 4+1 / 4,3 / 4+3 / 4+1 / 2+1 / 2+1 / 2$, $1 / 4+1 / 4+1 / 2+1 / 2+1 / 2$.

(8) $n=3 ; 2 / 3+2 / 3+2 / 3+2 / 3+1 / 3,1 / 3+1 / 3+1 / 3+1 / 3+2 / 3$.

(9) $n=2 ; 1 / 2+1 / 2+1 / 2+1 / 2+1 / 2+1 / 2+1 / 2+1 / 2$.

(10) $g^{\prime}=1, n=4$ and $1 / 2+1 / 2$.

(11) $g^{\prime}=1, n=3$ and $2 / 3+1 / 3$.

(12) $g^{\prime}=1, n=2$ and $1 / 2+1 / 2+1 / 2+1 / 2$.

(13) $g^{\prime}=2, n=2$ and $\Pi: \Sigma \rightarrow \Sigma^{\prime}$ is an unramified covering. 
Proof. The isotopy class of a periodic map is realized as an analytic cyclic automorphism of a Riemann surface ([Ni2], [B2], [Ke]). If $g \geqq 2$, we obtain the possibility for periodic maps in the above table by easy calculations using the conditions (i)-(iv) in 1.3. We will prove the existence of these maps in §4.4. The case (i) is classical.

Q.E.D.

2. Marked generalized quotient. In this section, we classify marked generalized quotient spaces of pseudo-periodic maps of bounded surfaces which we need in $\S 3$.

2.1. Let $f: \Sigma_{g} \rightarrow \Sigma_{g}$ be an orientation-preserving homeomorphism of a closed surface of genus $g$. We call $f$ a pseudo-periodic map if $f$ is isotopic to a homeomorphism $f^{\prime}: \Sigma_{g} \rightarrow \Sigma_{g}$ such that the following conditions are satisfied:

(i) There exists a disjoint union of simple closed curves $\mathcal{C}=C_{1} \cup C_{2} \cup \cdots \cup C_{r}$ on the interior of $\Sigma$ such that $f^{\prime}(\mathcal{C})=\mathcal{C}(\mathcal{C}$ might be empty).

(ii) Set $\mathcal{B}=\Sigma_{g}-\mathcal{C}$. Then the restriction $\left.f^{\prime}\right|_{\mathcal{B}}: \mathcal{B} \rightarrow \mathcal{B}$ is isotopic to a periodic map.

Note that a (non-periodic) pseudo-periodic map is said to be a surface transformation of algebraically finite type in Nielsen [Ni2], is said to be reducible with all component maps being of finite order in Thurston [T], and is said to be of parabolic type in Bers [B2].

We call $\mathcal{C}$ an admissible system of cut curves if each connected component of $\mathcal{B}$ has negative Euler number. If $\Sigma$ has negative Euler number, such a system always exists in the isotopy class of $f$. For each component $C_{i}$ of $\mathcal{C}$, there exists a minimal integer $\alpha_{i}$ such that $f^{\alpha_{i}}\left(\vec{C}_{i}\right)=\vec{C}_{i}$. There also exists a minimal integer $L_{i}$ such that $f^{L_{i}} \mid C_{i}$ is a Dehn twist of $e_{i}$ times $\left(e_{i} \in \boldsymbol{Z}\right.$ ). We set $s\left(C_{i}\right)=e_{i} \alpha_{i} / L_{i}$ and call it the screw number of $f$ at $C_{i}$ (cf. [Ni2]). $f$ is said to be of negative type if $s\left(C_{i}\right)<0$ for any $1 \leq i \leq r$.

The curve $C_{i}$ is said to be amphidrome if $\alpha_{i}$ is even and $f^{\alpha_{i} / 2}\left(\vec{C}_{i}\right)=-\vec{C}_{i}$, and nonamphidrome otherwise.

For a pseudo-periodic map of negative type $f$, Matsumoto-Montesinos [MM1], [MM2] defined the notion of minimal generalized quotient $\pi: \Sigma_{g} \rightarrow \mathcal{S}_{f}$ as follows: $\mathcal{S}_{f}$ is a numerical chorizo space in the sense of [MM1], [MM2], i.e., $\mathcal{S}_{f}$ is the underling topological space of a Riemann surface with nodes so that a multiplicity $m_{j}$ is attached to each component $\mathcal{S}_{f}^{(j)}$ of $\mathcal{S}_{f}$. The map $\pi$ is a pinched covering (or Bers' deformation [B1]) such that the covering degree $\pi^{-1}\left(\mathcal{S}_{f}^{(j)}\right) \rightarrow \mathcal{S}_{f}^{(j)}$ coincides with $m_{j}$. Moreover $\pi$ corresponds in a canonical way to a special representative in the isotopy class of $f$ which is called the superstandard form ([MM1, $\S 4, \S 5]$ ). We call $\mathcal{S}_{f}$ the generalized quotient space of $f$. Since the explicit construction of $\mathcal{S}_{f}$ is important in our argument, we summarize it;

Let $\Sigma_{g}=\mathcal{A} \cup \tilde{\mathcal{B}}$, where $\mathcal{A}$ is the union of annular neighborhood of the curve in $\mathcal{C}$ such that $f(\mathcal{A})=\mathcal{A}$, and $\tilde{\mathcal{B}}$ is the closure of $\Sigma_{g}-\mathcal{A}$ so that $\left.f\right|_{\tilde{\mathcal{B}}}: \tilde{\mathcal{B}} \rightarrow \tilde{\mathcal{B}}$ is periodic. Let $P$ be a branch point of the cyclic covering of disjoint union of bounded surfaces $\tilde{\mathcal{B}} \rightarrow \tilde{\mathcal{B}} /\left(\left.f\right|_{\tilde{\mathcal{B}}}\right)$, and let $(m, \lambda, \sigma)$ be the valency of $P$. By Euclidean algorithm, we obtain a sequence of integers $a_{0}>a_{1}>\cdots>a_{l}=1$ such that

(2.1.1) $\quad a_{0}=\lambda, \quad a_{1}=\sigma, \quad a_{j-1}+a_{j+1} \equiv 0\left(\bmod a_{j}\right) \quad(j=0,1, \ldots, l-1)$.

Set $m_{j}=m a_{j}(j=0,1, \ldots, l-1)$. Let $C(\tilde{\mathcal{B}})$ be the chorizo space constructed from 
$\tilde{\mathcal{B}} /\left(\left.f\right|_{\tilde{\mathcal{B}}}\right)$ by replacing a disk neighborhood of each branch point by a chain of a disk and $l$ spheres with multiplicities $m_{j}(0 \leq j \leq l)$. Note that, since the disk neighborhood $D_{P}$ of $P$ is oriented from the inside in [MM1], [MM2], they set $a_{0}=\lambda, a_{1}=\lambda-\sigma$. We orient $D_{P}$ from the outside as in $\$ 1.1$, so that we should put $a_{0}=\lambda, a_{1}=\sigma$.

Let $\mathcal{A}_{i} \subset \mathcal{A}$ be an annular neighborhood of $C_{i}$. Let $\left(m^{(1)}, \lambda^{(1)}, \sigma^{(1)}\right)$ and $\left(m^{(2)}, \lambda^{(2)}, \sigma^{(2)}\right)$ be the valencies of the boundary curves $C_{i}^{\prime}$ and $C_{i}^{\prime \prime}$ of $\mathcal{A}_{i}$, which are regarded as boundary curves of the periodic part $\tilde{\mathcal{B}}$.

We first assume $C_{i}$ to be non-amphidrome. Then $m^{(1)}=m^{(2)}=\alpha_{i}$, which we write $m$. We obtain a sequence of integers $a_{0}>a_{1}>\cdots>a_{u}=1$ and $b_{0}>b_{1}>\cdots>b_{v}=1$ such that

$$
\begin{aligned}
& a_{0}=\lambda^{(1)}, a_{1}=\sigma^{(1)}, a_{j-1}+a_{j+1} \equiv 0\left(\bmod a_{j}\right)(j=1,2, \ldots, u-1), \\
& b_{0}=\lambda^{(2)}, b_{1}=\sigma^{(2)}, b_{j-1}+b_{j+1} \equiv 0\left(\bmod b_{j}\right)(j=1,2, \ldots, v-1) .
\end{aligned}
$$

Set

$$
K=-s\left(C_{i}\right)-\delta^{(1)} / \lambda^{(1)}-\delta^{(2)} / \lambda^{(2)},
$$

where $\delta^{(j)}(j=1,2)$ are integers with $\sigma^{(j)} \delta^{(j)} \equiv 1\left(\bmod \lambda^{(j)}\right)$ and $0<\delta^{(j)}<\lambda^{(j)}$. Note that if $\lambda^{(j)}=1$, then we put $\delta^{(j)}=0 . K$ is an integer greater than or equal to -1 . Then the chorizo space $\operatorname{Ch}(\mathcal{A})$ is defined by a chain of two disks and several spheres whose multiplicities are as follows:

(i) If $K \geq 1$, then the multiplicities are

$$
(m a_{0}, m a_{1}, \ldots, m a_{u}, \underbrace{m, m, \ldots, m}_{K-1}, m b_{v}, \ldots, m b_{1}, m b_{0}) \text {. }
$$
$\left.m b_{0}\right)$.

(ii) If $K=0$, then the multiplicities are $\left(m a_{0}, m a_{1}, \ldots, m a_{u-1}, m, m b_{v-1}, \ldots, m b_{1}\right.$,

(iii) If $K=-1$, then we can find $u_{0}<u$ and $v_{0}<v$ so that $a_{u_{0}}=b_{v_{0}}$ and $\left(a_{u_{0}-1}+\right.$ $\left.b_{v_{0}-1}\right) / a_{u_{0}}$ is an integer greater than 1. Then the multiplicities are $\left(m a_{0}, m a_{1}, \ldots, m a_{u_{0}}\right.$, $\left.m b_{v_{0}-1}, \ldots, m b_{1}, m b_{0}\right)$.

Note that $m a_{0}$ and $m b_{0}$ are multiplicities of disks on both sides while the others are of spheres.

We next assume $C_{i}$ to be amphidrome. Then $C_{i}^{\prime}$ and $C_{i}^{\prime \prime}$ have the same valency $(2 m, \lambda, \sigma)$, where $2 m=\alpha_{i}$. Put $a_{0}=\lambda, a_{1}=\sigma, a_{0}>a_{1}>\cdots>a_{u}=1$ and $a_{j-1}+a_{j+1} \equiv 0\left(\bmod a_{j}\right)$ $(1 \leq j \leq u-1)$ similarly. Then $K=-s\left(C_{i}\right) / 2-\delta / \lambda$ is a non-negative integer where $\delta \sigma \equiv 1(\bmod \lambda)$. The chorizo space $\operatorname{Ch}(\mathcal{A})$ consists of a disk and $u+K+2$ spheres, whose dual graph is a Dynkin diagram of type $D$ and the multiplicities are

$$
(2 m a_{0}, 2 m a_{1}, \ldots 2 m a_{u}, \underbrace{2 m, \ldots, 2 m}_{K} \text { (the tree part), } m, m \text { (the terminal part)) }
$$

(see [MM2, p.73, Figure 3]).

Then $\operatorname{Ch}(\mathcal{B})$ and $\operatorname{Ch}(\mathcal{A})$ 's are naturally patched together and we obtain the space $\mathcal{S}_{f}$. Note that $\mathcal{S}_{f}$ is uniquely determined by the conjugacy class of $f$ ([MM1, §5]).

2.2. Let $\Sigma$ be a surface of genus $g$ with $k$ boundary curves $\partial_{1}, \ldots, \partial_{k}$. Let $f: \Sigma \rightarrow \Sigma$ be an orientation-preserving homeomorphism which satisfies (1) there is a disjoint union of 
simple closed curves $\mathcal{C}=\coprod_{j=1}^{r} C_{j}$ such that $\mathcal{C}$ and $\partial \Sigma=\coprod_{j=1}^{k} \partial_{j}$ do not intersect each other, (2) $\Sigma-\mathcal{C}$ is connected, (3) $f(\mathcal{C})=\mathcal{C}$ and $\left.f\right|_{\Sigma-\mathcal{C}}$ is periodic.

Let $\tilde{\Sigma}$ be the closed surface containing $\Sigma$ obtained by pasting disks $D_{j}(1 \leq j \leq k)$ along $\partial_{j}$ and let $\tilde{f}: \tilde{\Sigma} \rightarrow \tilde{\Sigma}$ be the extension of $f$ as in the proof of Lemma 1.2. For the map $\tilde{f}$, we construct a numerical chorizo space $S_{\tilde{f}}$ by the same method as in $\S 2.1$. Moreover, we add arrows at the top of the trees of $S_{\tilde{f}}$ corresponding to the center of the disks $D_{j}$ (see for instance (ii2) of Table 1). We also denote this space by $S_{f}$ and call it the marked generalized quotient space of $f$.

Now we classify $S_{f}$ for special values of $(g, r, k)$ and write the figures of $S_{f}$ in Table 1. We use the following notation. We write the valency data of $\partial_{j}$ by bold face characters. We enclose by double parentheses the valency data of the boundary curves $C_{j}^{\prime}$ and $C_{j}^{\prime \prime}$ of $\mathcal{A}_{j}$. If $f$ permutes the components of $\partial \Sigma$ or the components of $\mathcal{C}$ or the components of the boundary curves of $\mathcal{A}$, then we use the symbol of permutation. For example, if $f\left(\partial_{1}\right)=\partial_{2}, f\left(\partial_{2}\right)=\partial_{3}$ and $f\left(\partial_{3}\right)=\partial_{1}$, then we write $\left(\partial_{1}, \partial_{2}, \partial_{3}\right)$. If $C_{1}, \ldots, C_{s}(s \leq r)$ is amphidrome, we write $\operatorname{Amp}\left\{C_{1}, \ldots, C_{s}\right\}$. We denote the order of $f$ by $\operatorname{ord}(f)$, but we sometimes omit it if there is no fear of confusion.

(i) Assume $g=3$ and $r=k=0$. The map $\tilde{f}=f$ is one of the periodic maps classified in Lemma 1.4 (iii). Let $n, \sigma_{1} / \lambda_{1}+\cdots+\sigma_{l} / \lambda_{l}$ and $g^{\prime}$ be one of the data in Lemma 1.4 (iii). Then $\mathcal{S}_{f}$ has a star-shaped dual graph as in Figure 1(a). The component of the center of the graph has genus $g^{\prime}$ with multiplicity $n$ and has $l$ trees of spheres whose multiplicities $\left\{m_{11}, \ldots, m_{1 k_{1}}\right\}, \ldots,\left\{m_{l 1}, \ldots, m_{l k_{l}}\right\}$ are calculated by (2.1.1). From now on, we use the notation of (b) in Figure 1 instead of (a) for convenience. In order to describe $\mathcal{S}_{f}$ in each case of Lemma 1.4 (iii), we write the data $n, g^{\prime}$ and $\left\{m_{11}, \ldots, m_{1 k_{1}}\right\}, \ldots,\left\{m_{l 1}, \ldots, m_{l k_{k}}\right\}$ :

(1) $n=14,\{11,8,5,2,1\},\{10,6,2\},\{7\}$. (2) $n=14,\{3,1\},\{4,2\},\{7\}$.

(3) $n=14,\{13,12,11,10,9,8,7,6,5,4,3,2,1\},\{8,2\},\{7\}$. (4) $n=14,\{1\},\{6,4,2\},\{7\}$.

(5) $n=14,\{9,4,3,2,1\},\{12,10,8,6,4,2\},\{7\}$. (6) $n=14,\{5,1\},\{2\},\{7\}$.

(7) $n=12,\{11,10,9,8,7,6,5,4,3,2,1\},\{7,2,1\},\{6\}$. (8) $n=12,\{1\},\{5,3,1\},\{6\}$.

(9) $n=12,\{11,10,9,8,7,6,5,4,3,2,1\},\{9,6,3\},\{4\}$. (10) $n=12,\{1\},\{3\},\{8,4\}$.

(11) $n=12,\{7,2,1\},\{9,6,3\},\{8,4\} . \quad(12) n=12,\{5,3,1\},\{3\},\{4\}$.

(13) $n=9,\{8,7,6,5,4,3,2,1\},\{4,3,2,1\},\{6,3\}$. (14) $n=9,\{1\},\{5,1\},\{3\}$.

(15) $n=9,\{7,5,3,1\},\{5,1\},\{6,3\} . \quad(16) n=9,\{2,1\},\{4,3,2,1\},\{3\}$.

(17) $n=9,\{8,7,6,5,4,3,2,1\},\{7,5,3,1\},\{3\}$. (18) $n=9,\{1\},\{2,1\},\{6,3\}$.

(19) $n=8,\{7,6,5,4,3,2,1\},\{3,1\},\{6,4,2\} . \quad$ (20) $n=8,\{1\},\{5,2,1\},\{2\}$.

(21) $n=8,\{5,2,1\},\{5,2,1\},\{6,4,2\} . \quad(22) n=8,\{3,1\},\{3,1\},\{2\}$.

(23) $n=8,\{7,6,5,4,3,2,1\},\{7,6,5,4,3,2,1\},\{2\} . \quad$ (24) $n=8,\{1\},\{1\},\{6,4,2\}$.

(25) $n=7,\{6,5,4,3,2,1\},\{6,5,4,3,2,1\},\{2,1\} . \quad$ (26) $n=7,\{1\},\{1\},\{5,3,1\}$.

(27) $n=7,\{6,5,4,3,2,1\},\{5,3,1\},\{3,2,1\} . \quad$ (28) $n=7,\{1\},\{2,1\},\{4,1\}$.

(29) $n=7,\{6,5,4,3,2,1\},\{4,1\},\{4,1\}$. (30) $n=7,\{1\},\{3,2,1\},\{3,2,1\}$.

(31) $n=7,\{5,3,1\},\{5,3,1\},\{4,1\} . \quad$ (32) $n=7,\{2,1\},\{2,1\},\{3,2,1\}$.

(33) $n=6,\{5,4,3,2,1\},\{1\},\{3\},\{3\} . \quad$ (34) $n=6,\{5,4,3,2,1\},\{2\},\{2\},\{3\}$.

(35) $n=6,\{1\},\{4,2\},\{4,2\},\{3\} . \quad$ (36) $n=4,\{3,2,1\},\{3,2,1\},\{3,2,1\},\{3,2,1\}$. 


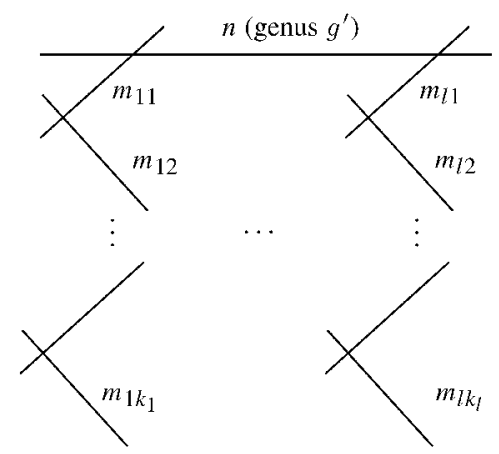

(a)

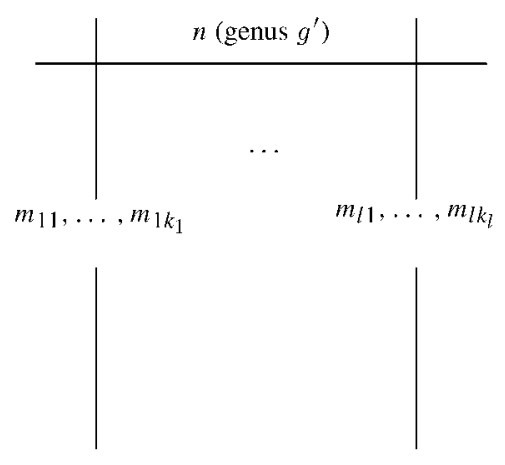

(b)

FIGURE 1.

(37) $n=4,\{1\},\{1\},\{1\},\{1\} . \quad$ (38) $n=4,\{3,2,1\},\{3,2,1\},\{1\},\{1\}$.

(39) $n=4,\{3,2,1\},\{3,2,1\},\{2\},\{2\},\{2\} . \quad$ (40) $n=4,\{1\},\{1\},\{2\},\{2\},\{2\}$.

(41) $n=3,\{2,1\},\{2,1\},\{2,1\},\{2,1\},\{1\} . \quad(42) n=3,\{1\},\{1\},\{1\},\{1\},\{2,1\}$.

(43) $n=2,\{1\},\{1\},\{1\},\{1\},\{1\},\{1\},\{1\},\{1\} . \quad$ (44) $n=4, g^{\prime}=1,\{2\},\{2\}$.

(45) $n=3, g^{\prime}=1,\{2,1\},\{1\} . \quad$ (46) $n=2, g^{\prime}=1,\{1\},\{1\},\{1\},\{1\}$.

(47) $n=2, g^{\prime}=2,\{\emptyset\}$.

(ii) Assume $g=2, r=0$ and $k=1 . \tilde{f}$ is one of the periodic maps classified in Lemma 1.4 (ii). Then the total valency of $\tilde{f}$ is one of the following:
(1) $\tilde{f}=\mathrm{id}_{\tilde{\Sigma}}$.
(2) $\mathbf{7} / \mathbf{1 0}+4 / 5+1 / 2$.
(5) $\mathbf{1} / \mathbf{1 0}+2 / 5+1 / 2$.
(3) $\mathbf{3} / \mathbf{1 0}+1 / 5+\mathbf{1} / 2$.
(4) $\mathbf{9} / \mathbf{1 0}+3 / 5+1 / 2$.
(8) $1 / 8+3 / 8+1 / 2$.
(6) $7 / 8+5 / 8+1 / 2$.
(7) $7 / 8+\mathbf{5} / \mathbf{8}+1 / 2$.
(11) $1 / 6+1 / 6+2 / 3$.
(9) $1 / 8+\mathbf{3} / \mathbf{8}+1 / 2$.
(10) $5 / 6+5 / 6+1 / 3$.
(14) $1 / 5+1 / 5+3 / 5$.
(12) $4 / 5+4 / 5+2 / 5$.
(13) $4 / 5+4 / 5+\mathbf{2} / \mathbf{5}$.
(17) $3 / 5+3 / 5+4 / 5$.
(15) $1 / 5+1 / 5+3 / 5$.
(16) $3 / 5+3 / 5+4 / 5$.
(20) $3 / 4+1 / 4+1 / 2+1 / 2$
(18) $2 / 5+2 / 5+1 / 5$.
(19) $2 / 5+2 / 5+\mathbf{1} / \mathbf{5}$.
(23) $2 / 3+2 / 3+1 / 3+1 / 3$.
(21) $3 / 4+1 / 4+1 / 2+1 / 2$.
(22) $2 / 3+2 / 3+1 / 3+1 / 3$.
(24) $1 / 2+1 / 2+1 / 2+1 / 2+1 / 2+1 / 2$.
(25) $1 / 2+1 / 2$. In this case, $g^{\prime}=1$ where $g^{\prime}$ is the genus of $\Sigma /\langle\tilde{f}\rangle$.

We write the graphs of $S_{f}$ of (ii), (1)-(25) in (ii1)-(ii25) of Table 1.

(iii) Assume $g=3, r=1$ and $k=0$. Suppose that $C_{1}$ is non-amphidrome. Then we may consider any two valencies of the fixed points of in the list of Lemma 1.4 (ii) as the valencies at $C_{1}^{\prime}$ and $C_{1}^{\prime \prime}$ for $f$. Then:
(1) $\left.f\right|_{\Sigma-\mathcal{C}}=$ id.
(2) $((7 / 8))+((5 / 8))+1 / 2$.
(3) $((3 / 8))+((1 / 8))+1 / 2$.
(4) $((5 / 6))+((5 / 6))+1 / 3$.
(5) $((1 / 6))+((1 / 6))+2 / 3$.
(6) $((4 / 5))+((4 / 5))+2 / 5$.
(7) $((4 / 5))+4 / 5+((2 / 5))$.
(8) $((1 / 5))+((1 / 5))+3 / 5$.
(9) $((1 / 5))+1 / 5+((3 / 5))$.
(10) $((3 / 5))+((3 / 5))+4 / 5$.
(11) $((3 / 5))+3 / 5+((4 / 5))$.
$(12)((2 / 5))+((2 / 5))+1 / 5$.
(13) $((2 / 5))+2 / 5+((1 / 5))$.
(14) $((3 / 4))+((1 / 4))+1 / 2+1 / 2$. 


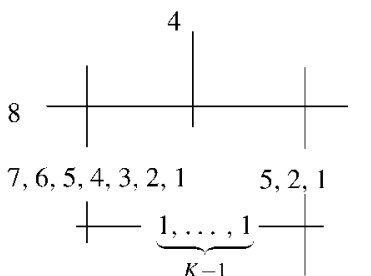

$K \geq 1$

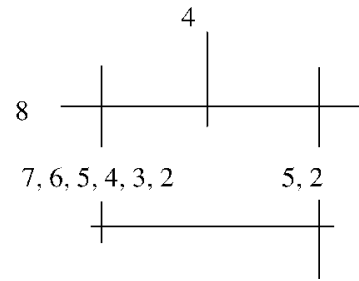

$K=0$

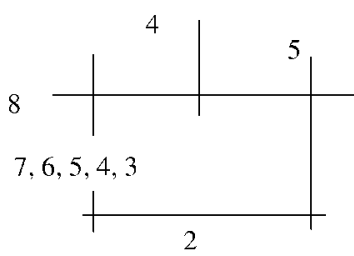

$K=-1$

FIGURE 2.

(15) $((2 / 3))+((2 / 3))+1 / 3+1 / 3 . \quad(16)((2 / 3))+2 / 3+((1 / 3))+1 / 3$.

(17) $((1 / 3))+((1 / 3))+2 / 3+2 / 3$. (18) $((1 / 2))+((1 / 2))+1 / 2+1 / 2+1 / 2+1 / 2$.

(19) $((1 / 2))+((1 / 2)), g^{\prime}=1$.

For instance, we consider the case of (2). Let $s\left(C_{1}\right)$ be the screw number at $C_{1}$ of $f$. The integer $K$ in (2.1.3) satisfies $K=-7 / 8-5 / 8-s\left(C_{1}\right) \geq-1$. Then $S_{f}$ is as in Figure 2 corresponding to $K \geq 1, K=0$ and $K=-1$.

If $K=-1$, then we first add the symbol " - " inside the sequence obtained by the algorithm (2.1.2), i.e., $8,7,6,5,4,3,2,1,-, 1,2,5,8$. Next we apply the "contraction" operations and "blow down" operations in [MM2, §6], and obtain 8, 7, 6, 5, 4, 3, 2, 5, 8.

Instead of these three graphs, we use the unified graph (iii2) in Table 1. In Table 1, the thick line means the tree of $K-1$ spheres $(K \geq 1)$, or means the identification of the multiplicity one components of the trees on both sides $(K=0)$, or means the result of shortening trees on both sides by the above two operations $(K=-1)$. The number beside the thick line means the multiplicity of each component of the tree.

Suppose $C_{1}$ is amphidrome. Then:

(20) $7 / 10+((4 / 5))+1 / 2 . \quad$ (21) $3 / 10+((1 / 5))+1 / 2 . \quad$ (22) $9 / 10+((3 / 5))+1 / 2$.

(23) $1 / 10+((2 / 5))+1 / 2$. (24) $5 / 6+5 / 6+((1 / 3))$. $\quad$ (25) $1 / 6+1 / 6+((2 / 3))$.

(26) $((1 / 3))+2 / 3+1 / 2+1 / 2$. (27) $1 / 3+((2 / 3))+1 / 2+1 / 2$.

(28) $3 / 4+1 / 4+((1 / 2))+1 / 2$.

(29) $\Sigma^{\prime} \rightarrow \Sigma^{\prime} /\langle\tilde{f}\rangle$ is a double covering with six branch points such that the disks $D_{1}^{\prime}$ and $D_{1}^{\prime \prime}$ do not contain any branch points, $f\left(C_{1}^{\prime}\right)=C_{2}^{\prime}$ and the valency at $C_{1}^{\prime}, C_{1}^{\prime \prime}$ is $(2,1,1)$. In this case, we write the total valency data as $1 / 2+1 / 2+1 / 2+1 / 2+1 / 2+1 / 2+((1))$. From now on, we use the same notation in a similar situation.

(30) $1 / 2+1 / 2+((1)), g^{\prime}=1$.

For instance, the graph $S_{f}$ of (20) is as in Figure 3. Instead of these two graphs, we use the unified graph (iii20) in Table 1.

(iv) Assume $g=1, r=0$ and $k=1$. Then:
(1) $f=$ id.
(2) $5 / 6+2 / 3+1 / 2$.
(4) $2 / 3+2 / 3+2 / 3$.
(5) $1 / 3+1 / 3+1 / 3$.
(3) $1 / 6+1 / 3+1 / 2$.
(7) $\mathbf{1 / 4}+1 / 4+1 / 2$.
(8) $1 / 2+1 / 2+1 / 2+1 / 2$.
(6) $3 / 4+3 / 4+1 / 2$. 


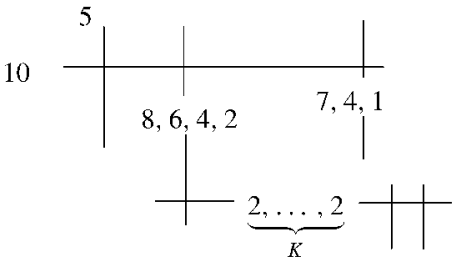

$K>0$

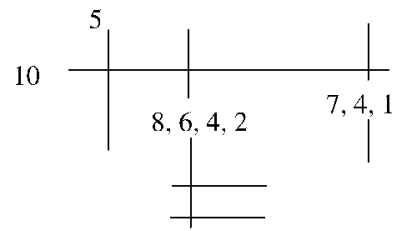

$K=0$

FIGURE 3.

(v) Assume $g=1, r=0$ and $k=2$.

(a) Suppose $f\left(\partial_{i}\right)=\partial_{i}(i=1,2)$. Then:

(1) $f=$ id. $\quad$ (2) $3 / 4+3 / 4+1 / 2$. (3) $1 / 4+1 / 4+1 / 2$.

(4) $2 / 3+2 / 3+2 / 3$. (5) $1 / 3+1 / 3+1 / 3$. (6) $1 / 2+1 / 2+1 / 2+1 / 2$.

(b) Suppose $\left(\partial_{1}, \partial_{2}\right)$. Then:

(1) $5 / 6+\mathbf{2} / \mathbf{3}+1 / 2$. (2) $1 / 6+\mathbf{1} / \mathbf{3}+1 / 2$.

(4) $1 / 4+1 / 4+\mathbf{1} / \mathbf{2}$. (5) $1 / 2+1 / 2+1 / 2+1 / 2+\mathbf{1}$.

(6) $f$ comes from an unramified double covering.

(vi) Assume $g=1, r=0$ and $k=3$.

(a) Suppose $f\left(\partial_{i}\right)=\partial_{i}(i=1,2,3)$. Then:

(1) $f=$ id. (2) $2 / 3+2 / 3+2 / 3$. (3) $1 / 3+1 / 3+1 / 3$. (4) $1 / 2+1 / 2+1 / 2+1 / 2$.

(b) Suppose $\left(\partial_{1}, \partial_{2}\right)$ and $f\left(\partial_{3}\right)=\partial_{3}$. Then:

(1) $5 / 6+2 / 3+1 / 2$. (2) $1 / 6+1 / 3+1 / 2$.

(4) $\mathbf{1} / \mathbf{4}+1 / 4+\mathbf{1} / \mathbf{2}$. (5) $\mathbf{1} / \mathbf{2}+1 / 2+1 / 2+1 / 2+\mathbf{1}$.

(3) $3 / 4+3 / 4+1 / 2$.

(c) Suppose $\left(\partial_{1}, \partial_{2}, \partial_{3}\right)$. Then:

(1) $5 / 6+2 / 3+\mathbf{1} / \mathbf{2}$. (2) $1 / 6+1 / 3+\mathbf{1} / \mathbf{2}$. (3) $2 / 3+2 / 3+2 / 3+1$.

(4) $1 / 3+1 / 3+1 / 3+1$. (5) $f$ comes from an unramified triple covering.

(vii) Assume $g=2, r=1$ and $k=1$. Suppose $C_{1}$ is non-amphidrome. Then:

(1) $\left.f\right|_{\Sigma-\mathcal{C}}=$ id. $\quad(2)((2 / 3))+((2 / 3))+\mathbf{2} / \mathbf{3} . \quad(3)((1 / 3))+((1 / 3))+\mathbf{1} / \mathbf{3}$.

(4) $((1 / 2))+((1 / 2))+\mathbf{1} / \mathbf{2}+1 / 2$.

Suppose $A m p\left\{C_{1}\right\}$. Then:

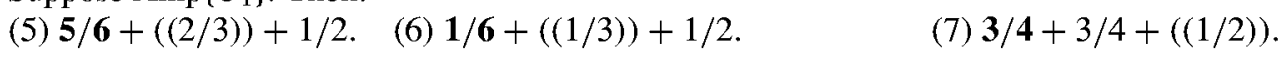

(8) $\mathbf{1} / \mathbf{4}+1 / 4+((1 / 2))$. (9) $\mathbf{1 / 2}+1 / 2+1 / 2+1 / 2+((1))$.

(viii) Assume $g=3, r=2$ and $k=0$. Then:

(1) $\left.f\right|_{\Sigma-\mathcal{C}}=$ id. (2) $((3 / 4))+((3 / 4))+((1 / 2)) . \quad(3)((1 / 4))+((1 / 4))+((1 / 2))$.

(4) $((1 / 2))+((1 / 2))+((1 / 2))+((1 / 2))$. (5) $((1 / 2))+((1 / 2))+1 / 2+1 / 2+((1))$.

(6) $1 / 2+1 / 2+1 / 2+1 / 2+((1))+((1))$.

(7) Amp $\left\{C_{1}, C_{2}\right\}$ and $f$ comes from an unramified double covering.

(8) $\left(C_{1}, C_{2}\right), 1 / 2+1 / 2+1 / 2+1 / 2+((1))+((1))$.

(9) $\left(C_{1}, C_{2}\right), \operatorname{Amp}\left\{C_{1}, C_{2}\right\}, 1 / 4+1 / 4+1 / 2+((1))+((1))$.

(10) $\left(C_{1}, C_{2}\right), \operatorname{Amp}\left\{C_{1}, C_{2}\right\}, 3 / 4+3 / 4+1 / 2+((1))+((1))$. 
(11) $\left(C_{1}, C_{2}\right)$ and $f$ comes from an unramified double covering.

(12) Amp $\left\{C_{1}, C_{2}\right\},\left(C_{1}, C_{2}\right)$ and $f$ comes from an unramified four-fold covering.

(ix) Assume $g=r=0$ and $k=3$. Then:
(a) $f=$ id.
(b) $\operatorname{ord}(f)=2,\left(\partial_{1}, \partial_{2}\right), f\left(\partial_{3}\right)=\partial_{3}, \mathbf{1} / \mathbf{2}+1 / 2+\mathbf{1}$.
(c) $\operatorname{ord}(f)=3,\left(\partial_{1}, \partial_{2}, \partial_{3}\right), 1 / 3+2 / 3+1$.

(x) Assume $g=r=0$ and $k=4$. Then:
(a) $f=$ id.
(b) $\operatorname{ord}(f)=2,\left(\partial_{1}, \partial_{2}\right), f\left(\partial_{i}\right)=\partial_{i}(i=3,4), \mathbf{1} / \mathbf{2}+\mathbf{1} / \mathbf{2}+\mathbf{1}$.
(c) $\operatorname{ord}(f)=2,\left(\partial_{1}, \partial_{2}\right),\left(\partial_{3}, \partial_{4}\right), 1 / 2+1 / 2+1+1$.
(d1) $\operatorname{ord}(f)=3,\left(\partial_{1}, \partial_{2}, \partial_{3}\right), f\left(\partial_{4}\right)=\partial_{4}, 2 / 3+1 / 3+1$.
(d2) $\operatorname{ord}(f)=3,\left(\partial_{1}, \partial_{2}, \partial_{3}\right), f\left(\partial_{4}\right)=\partial_{4}, 2 / 3+\mathbf{1} / \mathbf{3}+\mathbf{1}$.
(e) $\operatorname{ord}(f)=4,\left(\partial_{1}, \partial_{2}, \partial_{3}, \partial_{4}\right), 3 / 4+1 / 4+1$.

(xi) Assume $g=r=1$ and $k=1$. Then:

(1) $\left.f\right|_{\Sigma-\mathcal{C}}=$ id.

(2) $\operatorname{ord}(f)=2, \operatorname{Amp}\left\{C_{1}\right\}, \mathbf{1} / \mathbf{2}+1 / 2+((1))$.

(xii) Assume $g=r=1$ and $k=2$. Then;

(a1) $\left.f\right|_{\Sigma-\mathcal{C}}=\mathrm{id}$

(a2) $\operatorname{ord}(f)=2, \operatorname{Amp}\left\{C_{1}\right\}, \mathbf{1} / \mathbf{2}+\mathbf{1} / \mathbf{2}+((1))$.

(b1) $\operatorname{ord}(f)=2, \operatorname{Amp}\left\{C_{1}\right\},\left(\partial_{1}, \partial_{2}\right), 1 / 2+1 / 2+((1))+1$.

(b2) $\operatorname{ord}(f)=2,\left(\partial_{1}, \partial_{2}\right),((1 / 2))+((1 / 2))+1$.

(xiii) Assume $g=r=1$ and $k=3$. Then:

(a) $\left.f\right|_{\Sigma-\mathcal{C}}=$ id.

(b) $\operatorname{ord}(f)=2, \operatorname{Amp}\left\{C_{1}\right\}, \mathbf{1} / \mathbf{2}+1 / 2+\mathbf{1}+((1))$.

(c) $\operatorname{ord}(f)=3,\left(\partial_{1}, \partial_{2}, \partial_{3}\right),((1 / 3))+((2 / 3))+1$.

(xiv) Assume $g=r=2$ and $k=1$. Then:

(1) $\left.f\right|_{\Sigma-\mathcal{C}}=$ id.

(2) $\operatorname{ord}(f)=2, \operatorname{Amp}\left\{C_{1}, C_{2}\right\}, \mathbf{1} / \mathbf{2}+1 / 2+((1))+((1))$.

(3) $\operatorname{ord}(f)=2,\left(C_{1}, C_{2}\right), \mathbf{1} / \mathbf{2}+1 / 2+((1))+((1))$.

(4) $\operatorname{ord}(f)=4, \operatorname{Amp}\left\{C_{1}, C_{2}\right\},\left(C_{1}^{\prime}, C_{2}^{\prime}, C_{1}^{\prime \prime}, C_{2}^{\prime \prime}\right), \mathbf{3} / \mathbf{4}+1 / 4+((1))$.

(5) $\operatorname{ord}(f)=4, \operatorname{Amp}\left\{C_{1}, C_{2}\right\},\left(C_{1}^{\prime}, C_{2}^{\prime}, C_{1}^{\prime \prime}, C_{2}^{\prime \prime}\right), 3 / 4+\mathbf{1} / \mathbf{4}+((1))$.

(xv) Assume $g=r=3$ and $k=0$. Then:

(1) $\left.f\right|_{\Sigma-\mathcal{C}}=$ id.

(2) $\operatorname{ord}(f)=2, \operatorname{Amp}\left\{C_{1}, C_{2}\right\}, f\left(C_{3}\right)=C_{3},((1 / 2))+((1 / 2))+((1))+((1))$.

(3) $\operatorname{ord}(f)=2, \operatorname{Amp}\left\{C_{1}, C_{2}, C_{3}\right\}, 1 / 2+1 / 2+((1))+((1))+((1))$.

(4) $\operatorname{ord}(f)=2, \operatorname{Amp}\left\{C_{3}\right\},\left(C_{1}, C_{2}\right) 1 / 2+1 / 2+((1))+((1))+((1))$.

$(5) \operatorname{ord}(f)=2,\left(C_{1}, C_{2}\right), f\left(C_{3}\right)=C_{3},((1 / 2))+((1 / 2))+((1))+((1))$.

(6) $\operatorname{ord}(f)=3,\left(C_{1}, C_{2}, C_{3}\right), 1 / 3+2 / 3+((1))+((1))$.

(7) $\operatorname{ord}(f)=4, \operatorname{Amp}\left\{C_{1}, C_{2}\right\}, f\left(C_{3}\right)=C_{3},\left(C_{1}^{\prime}, C_{2}^{\prime}, C_{1}^{\prime \prime}, C_{2}^{\prime \prime}\right),((1 / 4))+((3 / 4))+((1))$.

(8) $\operatorname{ord}(f)=6, \operatorname{Amp}\left\{C_{1}, C_{2}, C_{3}\right\},\left(C_{1}^{\prime}, C_{2}^{\prime}, C_{3}^{\prime}, C_{1}^{\prime \prime}, C_{2}^{\prime \prime}, C_{3}^{\prime \prime}\right), 1 / 6+5 / 6+((1))$. 
3. Pseudo-Periodic maps of genus three. In this section, we classify the conjugacy classes of pseudo-periodic maps of negative type of genus three.

3.1. In order to classify decompositions of a Riemann surface $\Sigma_{3}=\mathcal{B} \cup \mathcal{C}$ of genus three by an admissible system $\mathcal{C}$ of cut curves, we introduce the weighted graphs A-O in Table 2. In Table 2, a vertex $v$ (which we express by a small circle for visual impression) corresponds to a connected component $B_{v}$ of $\mathcal{B}$. An edge corresponds to a component in $\mathcal{C}$ which is adjacent to two connected components of $\mathcal{B}$. We disregard the orientation of edges in $\$ 3.1$.

Let $g\left(B_{v}\right)$ be the genus of $B_{v}$ and let $\rho(v)$ be the number of those curves in $\mathcal{C}$ that are adjacent only to the component $B_{v}$. The number inside the small circle in Table 2 means $g\left(B_{v}\right)+\rho(v)$. We omit the number when it is zero. For instance, the graph (B) in Table 2 represents six types of decompositions, that is, the component corresponding to $v_{1}$ has genus $i_{1}$ and is adjacent to $2-i_{1}$ non-separating curves in $\mathcal{C}$, and the component corresponding to $v_{2}$ has genus $i_{2}$ and is adjacent to $1-i_{2}$ non-separating curves in $\mathcal{C}\left(0 \leq i_{1} \leq 2,0 \leq i_{2} \leq 1\right)$. We write it as $\mathrm{B}_{i_{1} i_{2}}$. For example, $\mathrm{B}_{01}$ represents the decomposition in Figure 4.

LEMMA 3.2. The decompositions of a Riemann surface of genus three by an admissible system of cut curves can be classified in terms of the following weighted graphs;

$\mathrm{A}_{3}, \mathrm{~A}_{2}, \mathrm{~A}_{1}, \mathrm{~A}_{0}, \mathrm{~B}_{21}, \mathrm{~B}_{20}, \mathrm{~B}_{11}, \mathrm{~B}_{10}, \mathrm{~B}_{01}, \mathrm{~B}_{00}, \mathrm{C}_{111}, \mathrm{C}_{110}, \mathrm{C}_{101}, \mathrm{C}_{001}, \mathrm{C}_{010}, \mathrm{C}_{000}, \mathrm{D}_{111}, \mathrm{D}_{110}$, $D_{001}, D_{000}, E_{11}, E_{10}, E_{00}, F_{11}, F_{10}, F_{00}, G_{11}, G_{10}, G_{01}, G_{00}, H_{1}, H_{0}, I_{1}, I_{0}, J_{1}, J_{0}, K_{1}, K_{0}, L$, $\mathrm{M}, \mathrm{N}, \mathrm{O}$.

Proof. By easy calculation. Note that this problem is equivalent to classifying stable curves of genus three, which is well-known. (See, for example, $[\mathrm{F}]$ ).

3.3. Let $X$ be one of the weighted graphs listed in Lemma 3.2. By an automorphism $\sigma: X \rightarrow X$, we mean an automorphism of the graph such that the weight $\left(g\left(\mathcal{B}_{v}\right), \rho(v)\right)$ coincides with $\left(g\left(\mathcal{B}_{\sigma(v)}\right), \rho(\sigma(v))\right)$ for each vertex $v$ of $X$.

In order to study the amphidrome action of a pseudo-periodic map, MatsumotoMontesinos [MMI, \$7] introduced the notion of "extended partition graph" of an admissible system of cut curves. In our case, we conveniently fix the orientation $\vec{e}$ of each edge $e$ in advance as in Table 2 and study the automorphisms of weighted graphs with their orientations taken into account.

For instance, consider the graph of type $\mathrm{E}_{11}$. We have three non-trivial automorphisms $\sigma$ as follows:

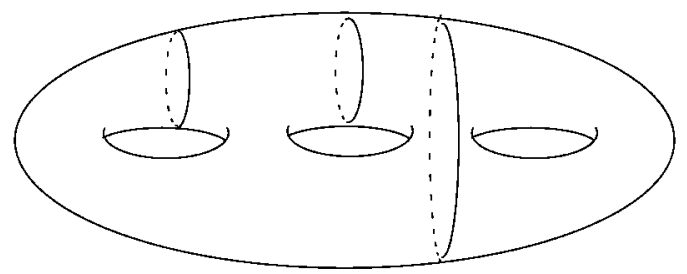

FIGURE 4 
(1) $\sigma$ fixes the vertices $v_{1}$ and $v_{2}$, and interchanges the edges $e_{1}$ and $e_{2}$ preserving their orientations.

(2) $\sigma$ interchanges the vertices $v_{1}$ and $v_{2}$ and fixes the edges $e_{1}$ and $e_{2}$ as a set, but changes their orientations.

(3) $\sigma$ interchanges the vertices $v_{1}$ and $v_{2}$ and interchanges the edges $e_{1}$ and $e_{2}$.

We express by $\left\langle\left(e_{1}, e_{2}\right)\right\rangle$ the cyclic group generated by the automorphism (1), express by $\left\langle\left(v_{1}, v_{2}\right)\left(e_{1},-e_{1}\right)\left(e_{2},-e_{2}\right)\right\rangle$ the cyclic group generated by the automorphism (2), and express by $\left\langle\left(v_{1}, v_{2}\right)\left(e_{1},-e_{2}\right)\right\rangle$ the cyclic group generated by the automorphism (3). This is equivalent to the following: We consider the symmetric group $S_{6}$ of formally independent six variables $v_{1}, v_{2}, e_{1},-e_{1}, e_{2},-e_{2}$. Then the group $\left\langle\left(e_{1}, e_{2}\right)\right\rangle$ is isomorphic to the subgroup of $S_{6}$ generated by the product $\left(e_{1}, e_{2}\right)\left(-e_{1},-e_{2}\right)$. The group $\left\langle\left(v_{1}, v_{2}\right)\left(e_{1},-e_{1}\right)\left(e_{2},-e_{2}\right)\right\rangle$ is isomorphic to the subgroup generated by the product $\left(v_{1}, v_{2}\right)\left(e_{1},-e_{1}\right)\left(e_{2},-e_{2}\right)$. The group $\left\langle\left(v_{1}, v_{2}\right)\left(e_{1},-e_{2}\right)\right\rangle$ is isomorphic to the subgroup generated by the product $\left(v_{1}, v_{2}\right)\left(e_{1},-e_{2}\right)$ $\left(e_{2},-e_{1}\right)$.

In order to express all cyclic automorphism groups of $X$, we introduce the subgroups $\mathrm{II}(0,1)-\mathrm{V}(1,1)$ of the symmetric group $\mathrm{S}_{17}$ of 17 variables $v_{1}, \ldots, v_{5}, \pm e_{1}, \ldots, \pm e_{6}$;

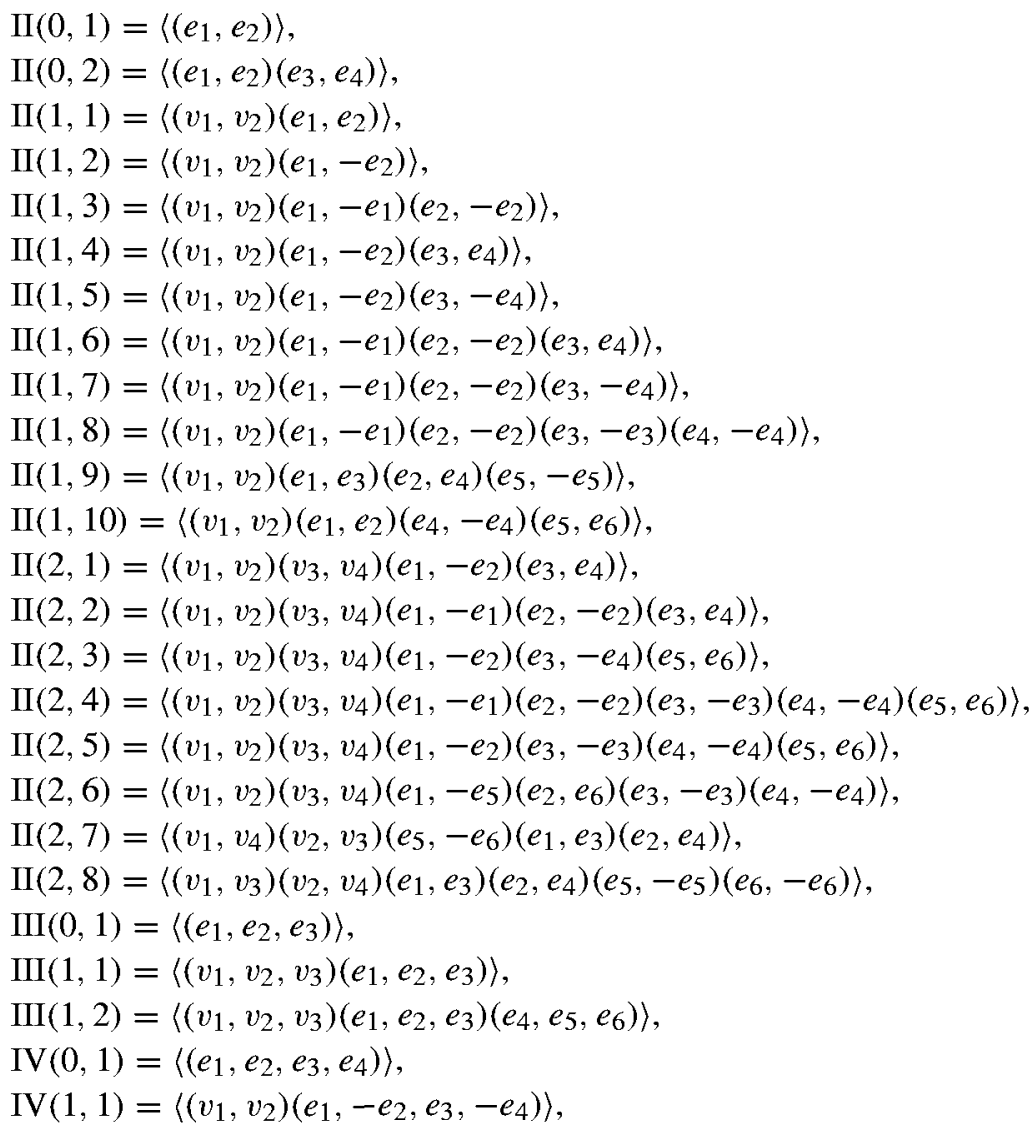




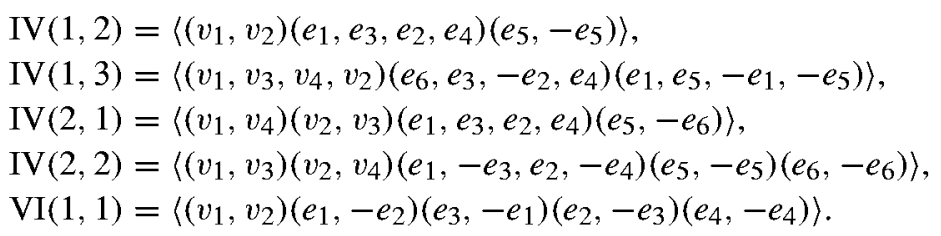

In the above table, for simplicity, we write $\left(\widetilde{e_{i_{1}}}, \ldots, \widetilde{e_{i_{l}}}\right)$ instead of $\left(\widetilde{e_{1}}, \ldots, \widetilde{e_{i_{l}}}\right)\left(-\widetilde{e_{i_{1}}}, \ldots\right.$, $\left.-\widetilde{e_{i_{l}}}\right)$, where $\widetilde{e_{i_{j}}}(j=1, \ldots, l)$ is one of $\pm e_{k}(k=1, \ldots, 6)$. The letters II, III, IV and VI mean that the order of the corresponding group is $2,3,4$ and 6 , respectively.

Suppose $X$ is of type $\mathrm{E}_{00}$ or $\mathrm{E}_{11}$. Then the non-trivial cyclic automorphism group of $X$ is $\mathrm{II}(0,1), \Pi(1,2)$ or $\mathrm{II}(1,3)$. For simplicity, we express this fact as

$$
\mathrm{E}_{i i}(0 \leq i \leq 1) \text { : } \mathrm{II}(0,1), \mathrm{II}(1,2), \mathrm{II}(1,3) .
$$

LEMMA 3.4. The non-trivial cyclic automorphism groups of the weighted graphs in Lemma 3.2 are classified as follows:

(1) $\mathrm{C}_{i i j}(0 \leq i, j \leq 1)$ : $\Pi(1,1)$.

(2) $\mathrm{D}_{i i j}(0 \leq i, j \leq 1): \mathrm{II}(1,1), \quad \mathrm{D}_{i i i}(0 \leq i \leq 1)$ : $\mathrm{III}(1,1)$.

(3) $\mathrm{E}_{i j}(0 \leq i, j \leq 1)$ : $\mathrm{II}(0,1), \quad \mathrm{E}_{i i}(0 \leq i \leq 1)$ : $\mathrm{II}(0,1), \mathrm{II}(1,2), \mathrm{II}(1,3)$.

(4) $\mathrm{F}_{i j}(0 \leq i, j \leq 1): \mathrm{I}(0,1), \quad \mathrm{F}_{i i}(0 \leq i \leq 1): \mathrm{\Pi}(0,1), \Pi(2,1), \mathrm{II}(2,2)$.

(5) $\mathrm{G}_{i j}(0 \leq i \leq 1)$ : II $(0,1)$.

(6) $\mathrm{H}_{i}(0 \leq i \leq 1)$ : $\mathrm{II}(0,1)$, $\mathrm{II}(0,1)$.

(7) $\mathrm{I}_{i}(0 \leq i \leq 1)$ : $\mathrm{I}(0,1), \mathrm{II}(0,1)$.

(8) $\mathrm{J}(0 \leq i \leq 1)$ : $\mathrm{II}(0,1), \mathrm{II}(1,4)$, $\mathrm{I}(1,6)$.

(9) $\mathrm{K}(0 \leq i \leq 1)$ : $\Pi(0,1), \Pi(1,4), \Pi(1,6)$.

(10) L: II $(0,1), \Pi(0,2), \mathrm{II}(1,5), \Pi(1,7), \mathrm{II}(1,8), \operatorname{III}(0,1), \operatorname{IV}(0,1), \operatorname{IV}(1,1)$, VI(1, 1).

(11) M: II $(0,1), \Pi(0,2), \operatorname{II}(1,9), \operatorname{IV}(1,2)$.

$(12) \mathrm{N}: \Pi(0,1), \Pi(0,2), \Pi(2,3), \Pi(2,4), \operatorname{II}(2,5), \Pi(2,7), \operatorname{II}(2,8), \operatorname{IV}(2,1), \operatorname{IV}(2,2)$.

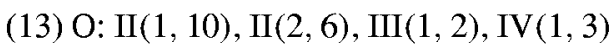

Elementary calculations are omitted.

3.5. Let $X$ and $G=\langle\sigma\rangle$ be a weighted graph and a cyclic automorphism group of $X$ described in Lemma 3.4. We define the quotient graph $Y$ of $X$ with respect to $G$ as follows (cf. [MM1, §7]): $Y$ is a weighted graph which may have loops and satisfies the following properties:

(i) There exists a map $h: X \rightarrow Y$ of graphs.

(ii) Let $|X|$ and $|Y|$ be the underling 1-dimensional cell complex of $X$ and $Y$, respectively. Then the map $h$ naturally induces a finite branched covering map $|h|:|X| \rightarrow|Y|$ such that the covering transformation group of $|h|$ coincides with $G$.

(iii) Let $\bar{v}$ be a vertex of $Y$. Then $h^{-1}(\bar{v})$ consists of a finite number, say $l(\bar{v})$, of vertices $v_{i}(1 \leq i \leq l(\bar{v}))$ such that their weights $\left(g\left(v_{i}\right), \rho\left(v_{i}\right)\right)$ coincide with each other, and denoted by $(g(\bar{v}), \rho(\bar{v}))$. In this sense, $\bar{v}$ has the triple weight $(l(\bar{v}), g(\bar{v}), \rho(\bar{v}))$.

(iv) Let $\bar{e}$ be an edge of $Y$. Then $h^{-1}(\bar{e})$ consists of a finite number, say $\xi(\bar{e})$, of edges of $X$. We put the weight $\xi(\bar{e})$ on $\bar{e}$. 
We remark that, if the vertices $v, v^{\prime}$ which are ends of an edge $e$ satisfies $h(v)=h\left(v^{\prime}\right)$, then $h(e)$ is a loop of $Y$ starting from and ending at $h(v)$.

Next we define the resolution $\tilde{Y}$ of $Y$. Suppose that there exists an edge $e$ of $X$ and an positive integer $m$ which satisfy $\sigma^{m}(e)=e, \sigma^{m}(v)=v^{\prime}$ and $\sigma^{m}\left(v^{\prime}\right)=v$, where $v$ and $v^{\prime}$ are the vertices at both ends of $e$. Let $\bar{m}$ be the minimal positive integer for $m$ which satisfy the above conditions. Then we replace $h(e)$ by a line with weight $2 \bar{m}$ where the top part is branched into two lines with weight $\bar{m}$ (see for instance Graph(6) in Table 3). We call the sum of these three lines $D$-edge of weight $2 \bar{m}$. After completing this process for every edge with the above property, we obtain a weighted graph $\tilde{Y}$, which we denote by $\tilde{Y}=X / G$.

In Table 3, the number inside a small circle (i.e., a vertex $\bar{v}$ ) means $g(\bar{v})+\rho(\bar{v})$, and the number beside the circle means $l(\bar{v})$. The number beside an edge $\bar{e}$ means $\xi(\bar{e})$. A loop is written with two arrows (see for instance Graph(5)). If $g(\bar{v})+\rho(\bar{v})=0$ or $l(\bar{v})=1$ or $\xi(\bar{e})=1$, then it is omitted. Note that one graph might represent several weighted graphs $\tilde{Y}$ as in the case of Table 2. Then we have:

LEMMA 3.6. For each weighted graph $X$ and each automorphism group $G$ of $X$ in Lemma 3.4, the resolution $X / G$ of quotient graph are as in Table 3 .

3.7. For a graph $\tilde{Y}=X / G$, we introduce the notion of substitution of marked generalized quotient in the following way: Since $\tilde{Y}$ is planer, we have an embedding $\iota: \tilde{Y} \hookrightarrow E^{2}$ into Euclidean plane $E^{2}$. We fix $\iota$. Let $v$ be a vertex of $\tilde{Y}$. Let $B(v, \varepsilon)$ be a closed ball of small radius $\varepsilon$ in $E^{2}$ with center $v$, and set

$$
V=\tilde{Y} \cap B(v, \varepsilon) .
$$

Suppose $e_{1}, \ldots, e_{s}, e_{s+1}, \ldots, e_{s+s^{\prime}}$ are the edges of $\tilde{Y}$ containing $v$ as end with each of $e_{s+1}, \ldots, e_{s+s^{\prime}}$ being a loop. Then $V$ consists of a vertex $v$ and $s+2 s^{\prime}$ segments $e_{1}^{\prime}, \ldots, e_{s}^{\prime}$, $e_{s+1}^{\prime}, e_{s+1}^{\prime \prime}, \ldots, e_{s+s^{\prime}}^{\prime}, e_{s+s^{\prime}}^{\prime \prime}\left(e_{i} \cap B(v, \varepsilon)=e_{i}^{\prime}\right.$ for $1 \leq i \leq s, e_{i} \cap B(v, \varepsilon)=e_{i}^{\prime} \cup e_{i}^{\prime \prime}$ for $s+1 \leq i \leq s+s^{\prime}$ ). Moreover, $V$ has a natural weighted graph structure induced by $\tilde{Y}$, i.e., the vertex $v$ has triple weight $(l(v), g(v), \rho(v))$ and the edge $e_{i}^{\prime}\left(1 \leq i \leq s+s^{\prime}\right)$ has weight $\xi\left(e_{i}\right)$, which the edge $e_{i}^{\prime \prime}\left(s+1 \leq i \leq s+s^{\prime}\right)$ has weight $\xi\left(e_{i}\right)$.

On the other hand, let $\Sigma$ be a surface of genus $g \leq 3$ with $k$ boundaries. Let $f: \Sigma \rightarrow \Sigma$ be one of the pseudo-periodic maps whose admissible system consists of $r$ curves classified in $\$ 2.2$, and satisfies the following conditions:

(i) $g=g(v), r=\rho(v)$ and $k=\left(\sum_{i=1}^{s+s^{\prime}} \xi\left(e_{i}^{\prime}\right)+\sum_{i=s+1}^{s+s^{\prime}} \xi\left(e_{i}^{\prime \prime}\right)\right) / l(v)$.

(ii) The chorizo space $S_{f}$ has $s+2 s^{\prime}$ arrows. Set $S_{f}=\sum_{j} m_{j} E_{j}+\sum_{i} n_{i} \vec{F}_{i}$, where $E_{j}$ is a component of $S_{f}$ and $\vec{F}_{i}$ is an arrow of $S_{f}\left(n_{i}, m_{j}\right.$ are their multiplicities). Changing the order if necessary, we have

$$
\xi\left(e_{i}^{\prime}\right)=l(v) n_{i}\left(1 \leq i \leq s+s^{\prime}\right), \quad \xi\left(e_{i}^{\prime \prime}\right)=l(v) n_{i}\left(s+s^{\prime}+1 \leq i \leq s+2 s^{\prime}\right) .
$$

We denote by $E\left(\vec{F}_{i}\right)$ the component of $\sum E_{j}$ which intersect $\vec{F}_{i}$. We substitute $S_{f}$ to $V$ in $\tilde{Y}$ in the following way: we replace the vertex $v$ by $\sum_{j} l(v) m_{j} E_{j}$ and connect each edge $e_{i}^{\prime}$ (or $\left.e_{i}^{\prime \prime}\right)$ to $\sum_{j} l(v) m_{j} E_{j}$ so that $e_{i}^{\prime}\left(\right.$ or $\left.e_{i}^{\prime \prime}\right)$ intersect $E\left(\vec{F}_{i}\right)$ transversally. 
We perform this process for each part $V$ of $\tilde{Y}$, and substitute each edge $e$ of $\tilde{Y}$ whose ends are vertices by trees of spheres. More precisely, this part of the graph has two or three types according as $K \geq 1, K=0$ (and $K=-1$ ) as we explained in $\$ 2.2$ (iii) Figure 2. We also substitute each $D$-edge by a tree of spheres of Dynkin diagram of type D (see [MM2, p. 73 Figure 3]). In this way, we obtain the generalized quotient space $S_{f}$ for a certain pseudoperiodic map $f: \Sigma_{3} \rightarrow \Sigma_{3}$ of negative type. The map $f$ has the following properties: Considering the types of vertices defined in Lemma 3.2, the graph $X$ naturally represents the admissible system of cut curves of $f$. The action to $X$ of $f$ coincides with $G$, and the stabilizer of $G$ for each connected component $B_{i}$ of $\Sigma_{3}-\mathcal{C}$ coincides with one of the periodic maps in $\$ 2.2$ whose marked generalized quotient space is just substituted to $V_{i}$ corresponding to $B_{i}$.

For instance, let $X=K_{1}$ and $G=\mathrm{II}(1,4)$. Then the parts of $K_{1} / \mathrm{II}(1,4)$ in Table $3(19)$ consist of

$$
\begin{aligned}
& V_{1}=\left\{v_{1}, e_{1}^{\prime} ; l\left(v_{1}\right)=g\left(v_{1}\right)=1, \rho\left(v_{1}\right)=0, \xi\left(e_{1}^{\prime}\right)=1\right\} \\
& V_{2}=\left\{v_{2}, e_{1}^{\prime}, e_{2}^{\prime} ; l\left(v_{2}\right)=1, g\left(v_{2}\right)=\rho\left(v_{2}\right)=0, \xi\left(e_{1}^{\prime}\right)=1, \xi\left(e_{2}^{\prime}\right)=2\right\} \\
& V_{3}=\left\{v_{3}, e_{2}^{\prime}, e_{3}^{\prime}, e_{3}^{\prime \prime} ; l\left(v_{3}\right)=2, g\left(v_{3}\right)=\rho\left(v_{3}\right)=0, \xi\left(e_{2}^{\prime}\right)=\xi\left(e_{3}^{\prime}\right)=\xi\left(e_{3}^{\prime \prime}\right)=2\right\} .
\end{aligned}
$$

By our rule, we substitute one of (iv1)-(iv8) in Table 1 to $V_{1}$, (ixb) to $V_{2}$ and (ixa) to $V_{3}$. We write this result as

$$
K_{1}: \mathrm{II}(1,4), \quad V_{1}=(\mathrm{iv}), \quad V_{2}=(\mathrm{ixb}), \quad V_{3}=(\mathrm{ixa}) .
$$

We have eight types of generalized quotient spaces in this case. For example, if we substitute $V_{1}=$ (iv2), then $S_{f}$ is as in Figure 5. Now we classify the conjugacy classes of the pseudoperiodic maps of negative type of genus three. By Matsumoto-Montesinos [MM2, Theorem 2], it is equivalent to classifying triples $\left(X, G, S_{f}\right)$ in our notation. We express $S_{f}$ by the substitution of the corresponding marked generalized quotients in Table 1 to the parts of the quotient graph $X / G$ in Table 2 or Table 3 . Since the substitution of (ixa) or (xa) is trivial, we omit it.

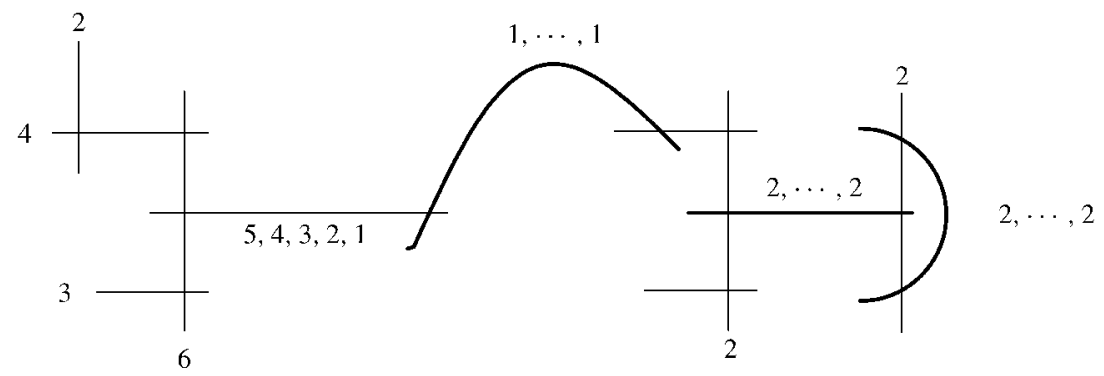

FIGURE 5. 
PROPOSITION 3.8. The conjugacy classes of the pseudo-periodic maps of negative type of genus three are classified as follows:

(1) $\mathrm{A}_{3}: \mathrm{Id}, V_{1}=$ (i) in $\$ 2.2$.

(2) $\mathrm{A}_{2}: \mathrm{Id}, V_{1}=$ (iii).

(3) $\mathrm{A}_{1}: \mathrm{Id}, V_{1}=$ (viii).

(4) $\mathrm{A}_{0}: \mathrm{Id}, V_{1}=(\mathrm{xv})$.

(5) $\mathrm{B}_{21}: \mathrm{Id}, V_{1}=$ (ii), $V_{2}=$ (iv).

(6) $\mathrm{B}_{20}:$ Id, $V_{1}=$ (ii), $V_{2}=$ (xi).

(7) $\mathrm{B}_{11}:$ Id, $V_{1}=$ (vii), $V_{2}=$ (iv).

(8) $\mathrm{B}_{10}: \mathrm{Id}, V_{1}=$ (vii), $V_{2}=$ (xi).

(9) $\mathrm{B}_{01}: \mathrm{Id}, V_{1}=$ (xiv), $V_{2}=$ (iv).

(10) $\mathrm{B}_{00}:$ Id, $V_{1}=$ (xiv), $V_{2}=$ (xi).

(11) $\mathrm{C}_{111}$ : Id, $V_{1}=V_{2}=$ (iv), $V_{3}=$ (va). $\Pi(1,1), V_{1}=$ (iv), $V_{2}=$ (vb).

(12) $\mathrm{C}_{110}$ : Id, $V_{1}=V_{2}=$ (iv), $V_{3}=$ (xiia). $\Pi(1,1), V_{1}=$ (iv), $V_{2}=$ (xiib).

(13) $\mathrm{C}_{101}:$ Id, $V_{1}=$ (iv), $V_{2}=$ (xi), $V_{3}=$ (va).

(14) $\mathrm{C}_{100}: \mathrm{Id}, V_{1}=$ (iv), $V_{2}=$ (xi), $V_{3}=$ (xiia).

(15) $\mathrm{C}_{001}$ : Id, $V_{1}=V_{2}=$ (xi), $V_{3}=$ (va). II $(1,1), V_{1}=$ (xi), $V_{2}=$ (vb).

(16) $\mathrm{C}_{000}$ : Id, $V_{1}=V_{2}=$ (xi), $V_{3}=$ (xiia). II $(1,1), V_{1}=$ (xi), $V_{2}=$ (xiib).

(17) $\mathrm{D}_{111}$ : Id, $V_{1}=V_{2}=V_{3}=$ (iv). II $(1,1), V_{1}=V_{3}=$ (iv), $V_{2}=(\mathrm{xb})$. $\operatorname{III}(1,1), V_{1}=$ (iv), $V_{2}=$ (ixc).

(18) $\mathrm{D}_{110}: \mathrm{Id}, V_{1}=V_{2}=$ (iv), $V_{3}=$ (xi). II $(1,1), V_{1}=$ (iv), $V_{2}=(\mathrm{xb}), V_{3}=$ (xi).

(19) $\mathrm{D}_{100}$ : Id, $V_{1}=$ (iv), $V_{2}=V_{3}=$ (xi). II $(1,1), V_{1}=$ (xi), $V_{2}=$ (xb), $V_{3}=$ (iv).

(20) $\mathrm{D}_{000}$ : Id, $V_{1}=V_{2}=V_{3}=$ (xi). II $(1,1), V_{1}=V_{3}=$ (xi), $V_{2}=$ (xb). $\mathrm{III}(1,1), V_{1}=$ (xi), $V_{2}=(\mathrm{ixc})$.

(21) $\mathrm{E}_{11}: \mathrm{Id}, V_{1}=V_{2}=$ (va). $\mathrm{II}(0,1), V_{1}=V_{2}=(\mathrm{vb}) . \quad \Pi(1,2), V_{1}=$ (va). $\mathrm{III}(1,3), V_{1}=$ (va).

(22) $\mathrm{E}_{10}:$ Id, $V_{1}=$ (va), $V_{2}=$ (viia). $\mathrm{II}(0,1), V_{1}=(\mathrm{vb}), V_{2}=$ (xiib).

(23) $\mathrm{E}_{00}$ : Id, $V_{1}=V_{2}=$ (viia). $\mathrm{II}(0,1), V_{1}=V_{2}=$ (viib). $\mathrm{II}(1,2), V_{1}=$ (xiia). $\operatorname{III}(1,3), V_{1}=$ (xiia).

(24) $\mathrm{F}_{11}$ : Id, $V_{1}=V_{2}=$ (iv). $\mathrm{II}(0,1), V_{1}=V_{4}=$ (iv), $V_{2}=V_{3}=$ (ixb). $\Pi(2,1), V_{1}=$ (iv). $\Pi(2,2), V_{1}=$ (iv).

(25) $\mathrm{F}_{10}$ : Id, $V_{1}=$ (iv), $V_{2}=$ (xi). II $(0,1), V_{1}=$ (iv), $V_{2}=V_{3}=$ (ixb), $V_{4}=$ (xi).

(26) $\mathrm{F}_{00}$ : Id, $V_{1}=V_{2}=$ (xi). $\mathrm{II}(0,1), V_{1}=V_{4}=(\mathrm{xi}), V_{2}=V_{3}=(\mathrm{ixb})$. $\mathrm{II}(2,1), V_{1}=(\mathrm{xi}) . \quad \mathrm{II}(2,2), V_{1}=(\mathrm{xi})$.

(27) $\mathrm{G}_{11}$ : Id, $V_{1}=$ (va), $V_{2}=$ (iv). II $(0,1), V_{1}=$ (vb), $V_{2}=$ (ixb), $V_{3}=$ (iv).

(28) $\mathrm{G}_{10}$ : Id, $V_{1}=$ (va), $V_{2}=$ (xi). II $(0,1), V_{1}=$ (vb), $V_{2}=(\mathrm{ixb}), V_{3}=$ (xi).

(29) $\mathrm{G}_{01}$ : Id, $V_{1}=$ (xiia), $V_{2}=$ (iv). II $(0,1), V_{1}=$ (xiib), $V_{2}=$ (ixb), $V_{3}=$ (iv).

(30) $\mathrm{G}_{00}$ : Id, $V_{1}=$ (xiia), $V_{2}=$ (xi). II $(0,1), V_{1}=$ (viib), $V_{2}=$ (ixb), $V_{3}=$ (xi).

(31) $\mathrm{H}_{1}$ : Id, $V_{1}=$ (via). $\mathrm{II}(0,1), V_{1}=\left(\right.$ vib), $V_{2}=(\mathrm{ixb})$. $\mathrm{III}(0,1), V_{1}=$ (vic),$V_{2}=(\mathrm{ixc})$. 
(32) $\mathrm{H}_{0}$ : Id, $V_{1}=$ (xiiia). II $(0,1), V_{1}=$ (xiiib), $V_{2}=(\mathrm{ixb})$. $\mathrm{III}(0,1), V_{1}=$ (xiiic), $V_{2}=$ (ixc).

(33) $\mathrm{I}_{1}$ : Id, $V_{1}=$ (iv). II $(0,1), V_{1}=(\mathrm{ixb}), V_{2}=(\mathrm{xb}), V_{3}=$ (iv). $\mathrm{III}(0,1), V_{1}=(\mathrm{ixc}), V_{2}=(\mathrm{xd}), V_{3}=(\mathrm{iv})$

(34) $\mathrm{I}_{0}$ : Id, $V_{1}=(\mathrm{xi}) . \quad \mathrm{II}(0,1), V_{1}=(\mathrm{ixb}), V_{2}=(\mathrm{xb}), V_{3}=(\mathrm{xi})$. $\mathrm{III}(0,1), V_{1}=(\mathrm{ixc}), V_{2}=(\mathrm{xd}), V_{3}=(\mathrm{xi})$.

(35) $\mathrm{J}_{1}$ : Id, $V_{3}=(\mathrm{va}) . \quad \mathrm{II}(0,1), V_{1}=(\mathrm{va}), V_{2}=V_{3}=$ (ixb). $\Pi(1,4), V_{1}=(\mathrm{vb}) . \quad \mathrm{II}(1,6), V_{1}=(\mathrm{vb})$.

(36) $\mathrm{J}_{0}$ : Id, $V_{3}=$ (xiia). $\Pi(0,1), V_{1}=$ (xiia), $V_{2}=V_{3}=$ (ixb). $\mathrm{II}(1,4), V_{1}=$ (xiib). II $(1,6), V_{1}=$ (xiib).

(37) $\mathrm{K}_{1}$ : Id, $V_{4}=$ (vi). II $(0,1), V_{1}=V_{2}=$ (ixb), $V_{4}=$ (iv). $\Pi(1,4), V_{1}=$ (iv), $V_{2}=$ (ixb). $\Pi(1,6), V_{1}=$ (iv), $V_{2}=(\mathrm{ixb})$.

(38) $\mathrm{K}_{0}$ : Id, $V_{4}=(\mathrm{xi}) . \quad \mathrm{II}(0,1), V_{1}=V_{2}=(\mathrm{ixb}), V_{4}=(\mathrm{xi})$. $\mathrm{II}(1,4), V_{1}=(\mathrm{xi}), V_{2}=(\mathrm{ixb}) . \quad \mathrm{II}(1,6), V_{1}=(\mathrm{xi}), V_{2}=(\mathrm{ixb})$.

(39) L: Id. $\quad I(0,1), V_{1}=V_{2}=(x b) . \quad \Pi(0,2), V_{1}=V_{2}=(x c) . \quad \Pi(1,5) . \quad I I(1,8)$. $\operatorname{III}(0,1), V_{1}=V_{2}=(\mathrm{xd}) . \quad \mathrm{IV}(0,1), V_{1}=V_{2}=(\mathrm{xe})$. $\operatorname{IV}(1,1), V_{1}=(\mathrm{xc}) . \quad \mathrm{VI}(1,1), V_{1}=(\mathrm{xd})$.

(40) M: Id. II $(0,1), V_{1}=(\mathrm{ixb}), V_{3}=(\mathrm{xb}) . \quad \mathrm{II}(0,2), V_{1}=(\mathrm{xc}), V_{2}=V_{3}=(\mathrm{ixb})$. $\Pi(1,9), V_{1}=(\mathrm{xc}) . \quad \mathrm{IV}(1,2), V_{1}=(\mathrm{xe}), V_{2}=(\mathrm{ixb})$.

(41) N: Id. $\operatorname{II}(0,1), V_{1}=V_{3}=(\mathrm{ixb}) . \quad \mathrm{II}(0,2), V_{1}=V_{2}=V_{3}=V_{4}=(\mathrm{ixb})$. $\operatorname{IV}(0,1) . \quad \Pi(2,4) . \quad \mathrm{II}(2,5) . \quad \Pi(2,7) . \quad \Pi(2,8)$. $\operatorname{IV}(2,1), V_{1}=V_{2}=(\mathrm{ixb}) . \quad \operatorname{IV}(2,2), V_{1}=V_{2}=(\mathrm{ixb})$.

(42) O: Id. II $(1,10), V_{1}=V_{2}=$ (ixb). $\quad \mathrm{II}(2,6) . \quad \mathrm{III}(1,2), V_{1}=$ (ixc). $\operatorname{IV}(1,3)$.

PROOF. Cyclic automorphisms of the weighted graph in Lemma 3.4 are clearly induced by pseudo-periodic maps of negative type. Therefore we get the assertion by easy calculations.

\section{Classification of degenerations.}

4.1. Let $\phi: S \rightarrow \Delta$ be a proper surjective holomorphic map to the unit disk $\Delta=\{t \in$ $C|| t \mid<1\}$ such that $\phi^{-1}(t)$ is a smooth Riemann surface of genus $g \geq 2$ for any $t \in \Delta^{*}=$ $\Delta-\{0\}$. We call $\phi$ a degeneration of genus $g$, and call $F=\phi^{-1}(0)$ the singular fiber. Suppose that $\phi$ is normally minimal, i.e., the reduced scheme of $F$ has normal crossing and any $(-1)$ curve in $F$ intersects the other components at at least three points. For two degenerations $\phi$ : $S \rightarrow \Delta$ and $\phi^{\prime}: S^{\prime} \rightarrow \Delta$, if there is an orientation-preserving homeomorphism $\psi: S \rightarrow S^{\prime}$ which satisfies $\phi^{\prime} \circ \psi=\phi$, then we say $\phi$ and $\phi^{\prime}$ are topologically equivalent.

Let $\boldsymbol{M}_{g}$ be the moduli space of Riemann surfaces of genus $g$ and let $\overline{\boldsymbol{M}}_{g}$ be the DeligneMumford compactification of $\boldsymbol{M}_{g}$ (cf. [DM]). Since $\Delta$ is complex one-dimensional, the moduli map $\mu: \Delta^{*} \rightarrow \boldsymbol{M}_{g}$ is uniquely extended to a morphism $\bar{\mu}: \Delta \rightarrow \overline{\boldsymbol{M}}_{g}$. We call the stable Riemann surface $\bar{\mu}(0)$ the moduli point of $\phi$.

By fixing $t_{0} \in \Delta^{*}$ as a base point, the canonical generator of $\pi\left(\Delta^{*}, t_{0}\right) \simeq Z$ acts naturally on the Riemann surface $\phi^{-1}\left(t_{0}\right)$ as an orientation-preserving homeomorphism modulo isotopy. Since a change of the base point corresponds to a conjugation in the mapping class 
group $\Gamma_{g}$, the degeneration of $\phi$ uniquely determines an element $m(\phi)$ of the conjugacy class $\hat{\Gamma}_{g}$ of $\Gamma_{g}$. We call $m(\phi)$ the topological monodromy of $\phi$. Then Matsumoto-Montesinos' fundamental theorem [MM2, Theorem 1] says the following:

An element of $\hat{\Gamma}_{g}$ which is the topological monodromy of a degeneration is represented by a pseudo-periodic map of negative type. Conversely, any conjugacy class of a pseudoperiodic map of negative type is realized as the topological monodromy of a certain degeneration. The topological monodromy uniquely determines the equivalence class of a topological type of a degeneration.

Thanks to this theorem, we can classify topological equivalence classes of degenerations of Riemann surfaces of genus three.

Let $f: \Sigma_{3} \rightarrow \Sigma_{3}$ be a pseudo-periodic map of negative type, and let $\phi_{f}: S \rightarrow \Delta$ be the degeneration whose topological monodromy coincides with the conjugacy class of $f$ (cf. [MM1, §10]). By the construction of $\phi_{f}$, the singular fiber $\phi_{f}^{-1}(0)$ coincides topologically with the generalized quotient space $S_{f}$ of $f$. Let $\mathcal{C}$ be the admissible system of cut curves of the class $f$. By shrinking each curve of $\mathcal{C}$ to a point, we naturally associate a stable Riemann surface $X_{f}$. In this sense, the weighted graphs classified in Lemma 3.2 (Table 2) can also be considered as the dual graphs of stable Riemann surfaces. Namely, a vertex of the graph corresponds to an irreducible component of $X_{f}$, the weights of the vertex are the genus and the number of double points of the component, and an edge corresponds to a double point of $X_{f}$ and so on.

Lemma 4.2. The topological structure of the stable Riemann surface which is the moduli point of $\phi_{f}: S \rightarrow \Delta_{\varepsilon}$ coincides with $X_{f}$.

Proof. Let $\mathcal{A}$ be an annular neighborhood of the admissible system $\mathcal{C}$. Since $f$ is pseudo-periodic, there exists a natural number $N$ so that $f^{N}$ can be considered (modulo isotopy) to be the identity map on $\Sigma_{3}-\mathcal{A}$ and $f^{N}$ behaves as an integral Dehn twist on each component of $\mathcal{A}$.

Now let $h: \Delta \rightarrow \Delta$ be the cyclic covering map $t \mapsto t^{N}$ between disks and let $\tilde{S}$ be the minimal desingularization of the fiber product $S \times \Delta \Delta$ via $h$. The natural map $\tilde{\phi}: \tilde{S} \rightarrow \Delta$ is a degeneration with singular fiber $\tilde{F}=\tilde{\phi}^{-1}(0)$ and the topological monodromy of $\tilde{\phi}$ coincides with $f^{N}$. From the construction of the chorizo space, $\tilde{F}$ is a semi-stable Riemann surface obtained from $X_{f}$ by replacing each double point of $X_{f}$ by a tree of smooth rational curves of multiplicity one. In other words, $\tilde{\phi}$ is the semi-stable reduction of $\phi_{f}$ and the stable model of $\tilde{F}$ coincides with $X_{f}$.

Hence the topological structure of the moduli point of $\phi_{f}$ coincides with $X_{f}$. Q.E.D.

By the previous arguments, we have:

THEOREM 4.3. Let $\phi: S \rightarrow \Delta$ be a degeneration of Riemann surfaces of genus three. Let $\bar{\mu}_{\phi}(0)^{\text {top }}$ be the topological structure of the stable Riemann surface associated to $\bar{\mu}_{\phi}(0)$. Let $m(\phi)$ and $F$ be the topological monodromy and the singular fiber of $\phi$, respectively. Then $\left(\bar{\mu}_{\phi}(0)^{\text {top }}, m(\phi), F\right)$ is one of the following:

(i) $\bar{\mu}_{\phi}(0)^{\text {top }}$ is one of those listed in Lemma 3.2. 
(ii) $m(\phi)$ is one of those listed in Proposition 3.8 associated with $\bar{\mu}_{\phi}(0)^{\mathrm{top}}$.

(iii) The topological structure of $F$ coincides with the generalized quotient space $S_{m(\phi)}$ which we described in Proposition 3.8.

Conversely, the degenerations which satisfy the properties (i), (ii), (iii) exist.

4.4. Lastly, we prove the latter part of Lemma 1.4 which we postponed in $\S 1$.

Step 1. Assume $g^{\prime} \geq 1$. If we fix $n, g^{\prime} \geq 1$ and $\left(\lambda_{1}, \ldots, \lambda_{l}\right)$, then the total valency is unique from the list of Lemma 1.4. Therefore the existence of the desired periodic map is clear by Harvey's theorem. Hence from now on, we assume $g^{\prime}=0$.

Step 2. From the data $n$ and $\left\{\sigma_{i} / \lambda_{i}\right\}_{1 \leq i \leq l}$, we obtain a sequence of integers $a_{0}^{i}>a_{1}^{i}>$ $\cdots>a_{k_{i}}^{i}$ which satisfy

$$
a_{0}^{i}=\lambda_{i}, \quad a_{1}^{i}=\sigma_{i}, \quad a_{j-1}^{i}+a_{j+1}^{i} \equiv 0 \quad\left(\bmod a_{j}^{i}\right) \quad\left(j=0,1, \ldots, k_{i}-1\right) .
$$

Set

$$
X=n E_{0}+\sum_{i=1}^{l} \sum_{j=1}^{k_{i}} \frac{n a_{j}^{i}}{\lambda_{i}} E_{j}^{i},
$$

where $E_{0} E_{1}^{i}=1, E_{0} E_{j}^{i}=0(j \geq 2), E_{j}^{i} E_{j+1}^{i}=1\left(j=1, \ldots, k_{i}-1\right), E_{j}^{i} E_{j^{\prime}}^{i^{\prime}}=0$ $\left(\left|j-j^{\prime}\right| \geq 2\right)\left(i, i^{\prime}=1, \ldots, l\right)$ and $E_{0}, E_{j}^{i}$ are nonsingular rational curves. $X$ naturally has the structure of a scheme.

By Winters [Wi, Cor. 4.3], there exist a nonsingular surface $\tilde{S}$ and a surjective holomorphic map $\tilde{\phi}: \tilde{S} \rightarrow \Delta$ such that $X$ is the fiber over the origin. We claim that any fiber of $\tilde{\phi}$ is connected. By [Wi, Cor. 3.7] it suffices to prove

$$
\operatorname{gcd}\left(n, n / \lambda_{1} a_{1}^{1}, \ldots, n / \lambda_{l} a_{1}^{l}\right)=1 .
$$

Suppose $\operatorname{gcd}\left(n \sigma_{1} / \lambda_{1}, \ldots, n \sigma_{l} / \lambda_{l}, n\right)=d \neq 1$. Then $n \sigma_{i} /\left(\lambda_{i} d\right)(1 \leq i \leq l)$ are integers. Since $\operatorname{gcd}\left(\sigma_{i}, \lambda_{i}\right)=1, \lambda_{i}$ divides $n / d$ for any $i(1 \leq i \leq l)$. This contradicts the condition $n=\operatorname{lcm}\left(\lambda_{1}, \ldots, \lambda_{l}\right)(\$ 1.3$ (iv) (2)).

Step 3. An irreducible component of a singular fiber of a certain degeneration is called main component when its genus is more than zero or it intersects the other components at more than two points.

Now we claim that the monodromy $\tilde{f}$ of the degeneration $\tilde{\phi}$ is periodic. Assume that $\tilde{f}$ has a non-empty admissible system $\mathcal{C}$. First assume that the number of connected components of $\Sigma_{g}-\mathcal{C}$ is one. If $\tilde{f}$ has no amphidrome, the dual graph of the chorizo space $S_{\tilde{f}}$ has loops. If $\tilde{f}$ has amphidrome, $S_{\tilde{f}}$ clearly has at least two main components. Since $X$ has only one main component $E_{0}$, this is a contradiction.

Next assume that the number $n$ of connected components of $\Sigma_{g}-\mathcal{C}$ is greater than one. Since $X$ has only one main component, the action on the dual graph induced by $\tilde{f}$ cyclically permutes all components. Then the quotient graph has a loop or $\tilde{f}$ has an amphidrome action at a certain part, which is a contradiction. Hence $\tilde{f}$ is periodic.

Considering multiplicities of the components of $X$, the valency data of the periodic map $\tilde{f}$ coincides with $\left\{\sigma_{i} / \lambda_{i}\right\}_{1 \leq i \leq k}$.

Q.E.D. 
TABLE 1. Marked generalized quotient.

(ii1)
$\downarrow$

(ii5)

(ii9)

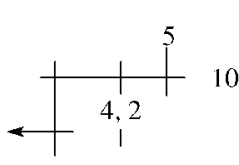

(ii2)

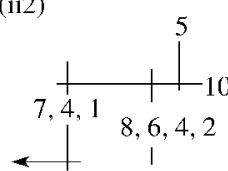

(ii6)

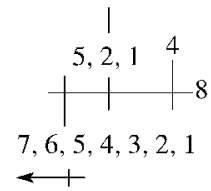

(ii10)

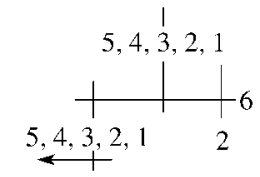

(ii 14)

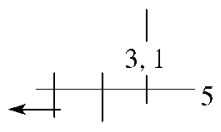

(ii18)

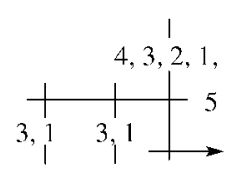

(ii21)
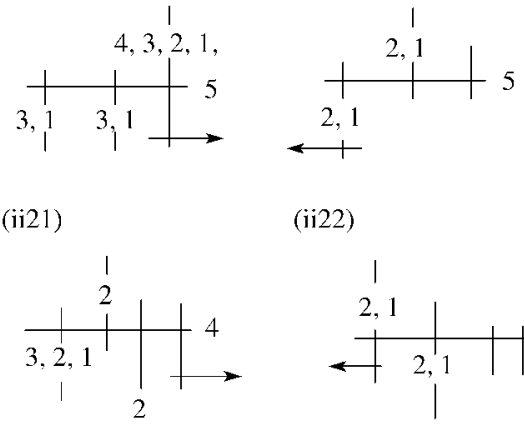

(ii22)

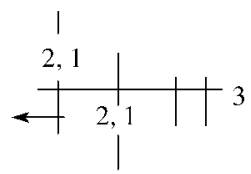

(ii3)

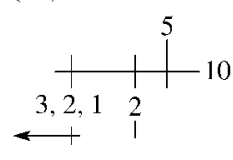

(ii7)

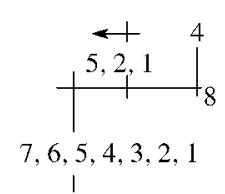

(ii11)

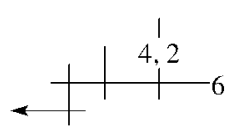

(ii15) (ii4)

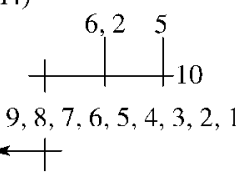

(ii8)

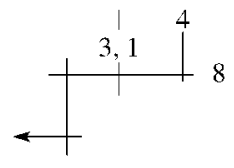

(iil2)

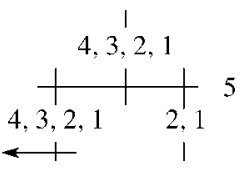

(ii16)

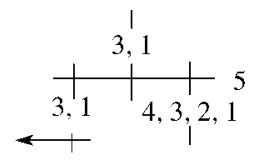

(ii20)
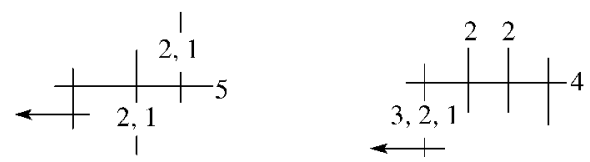

(ii24)

(ii23)

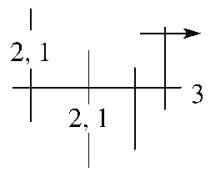

(ii25)

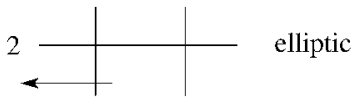


TABLE 1 (continued)
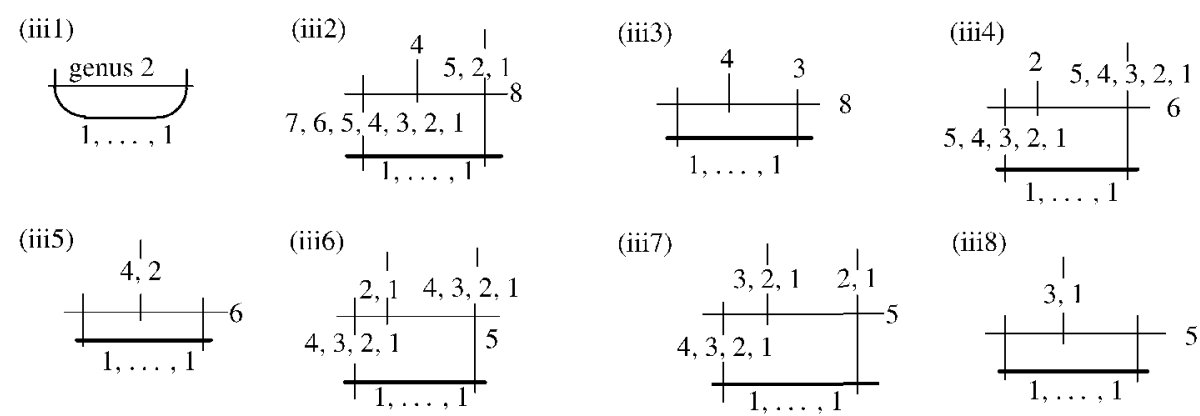

(iii7)

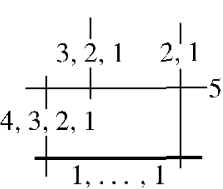

(iiis)
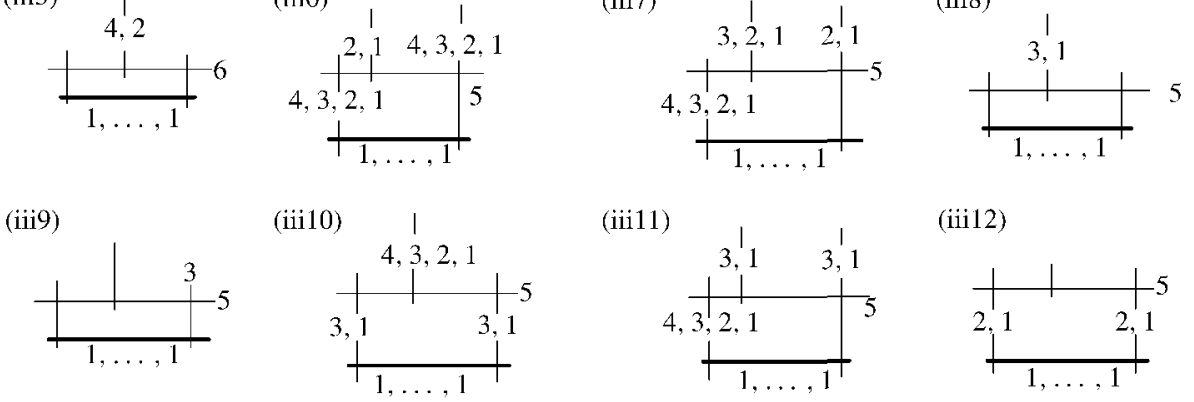

(iii11)

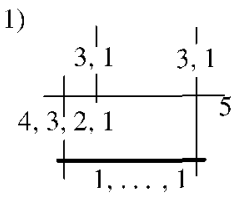

(iii12)

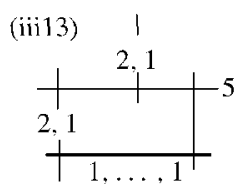

(iii)

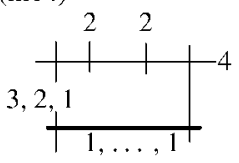

(iii15)
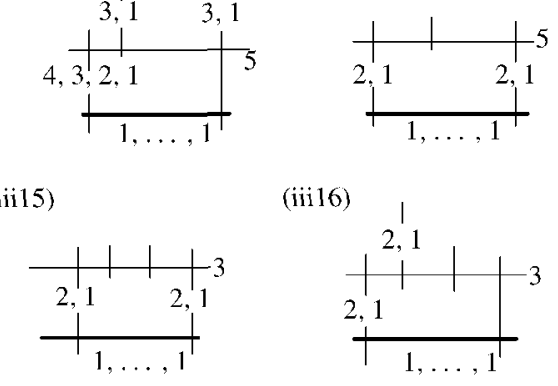

(iii16)
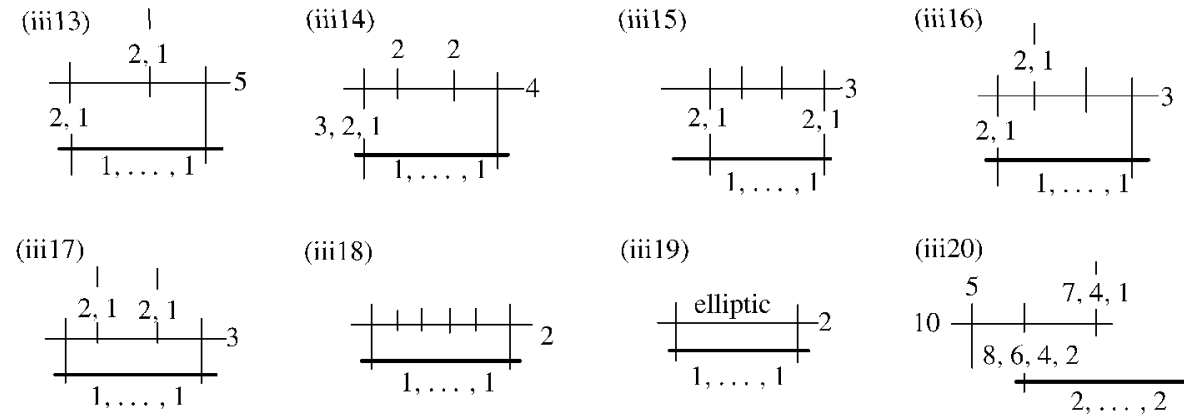

(iii) 8 )

(iii19)
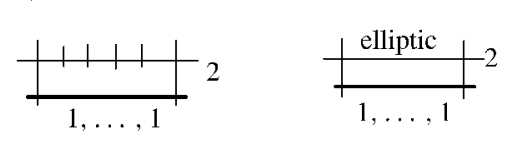

(iii20)

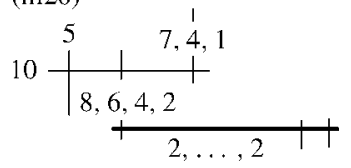

(iii21)

(iii22)

(iii23)

(iii24)

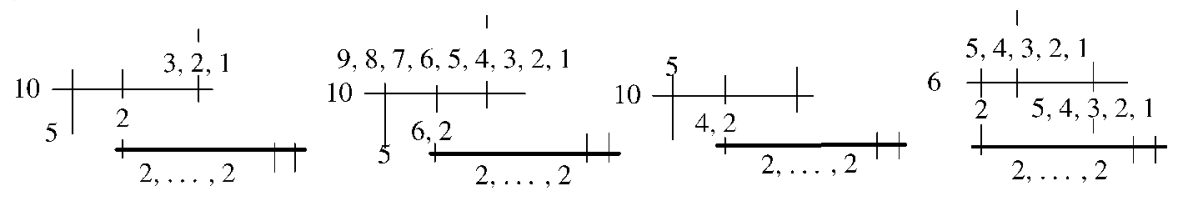

(iii25)

6

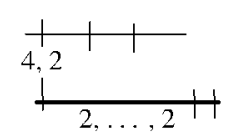

(iii29)

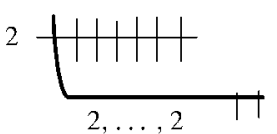

(iii26)

(iii27)
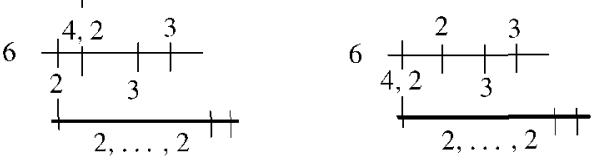

(iii28)

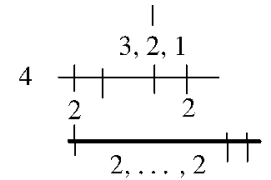

(iii30)

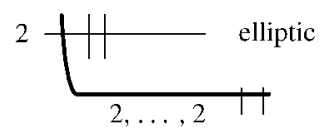


TABLE 1 (continued)

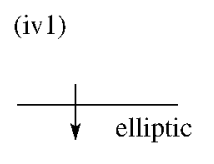

(iv5)

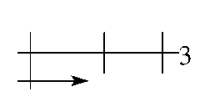

(val)

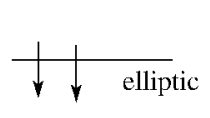

(va5)

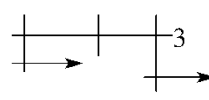

(vb3)

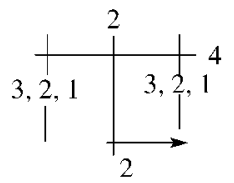

(vial)

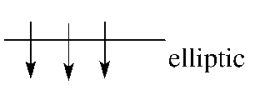

(vib1)

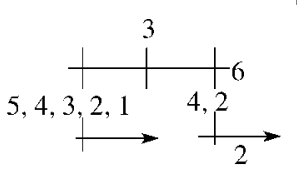

(vib2)

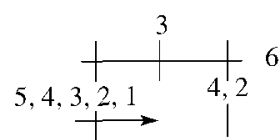

(iv6)

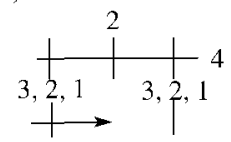

(va2)

(va6)

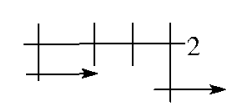

(vb4)

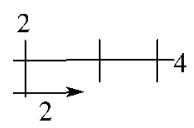

(via2)

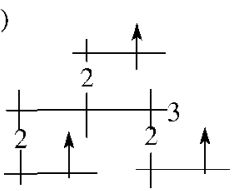

(vib2)
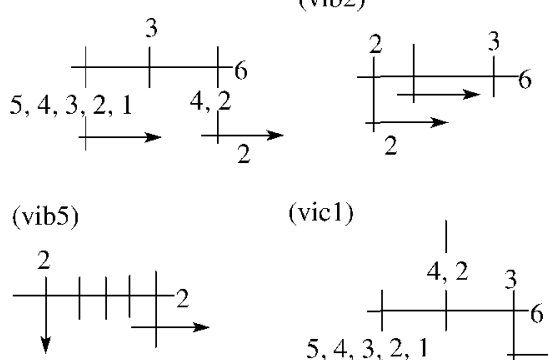

(vic4)

(vic1)

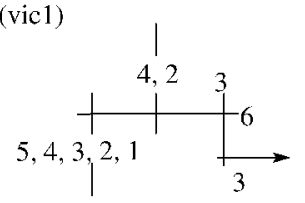

(vic5)

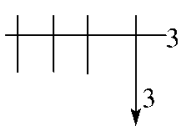

(iv3)

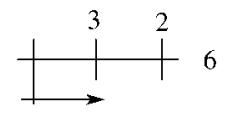

(iv7)

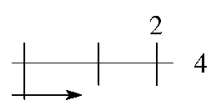

(va3)

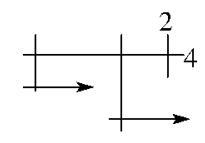

(vb1)

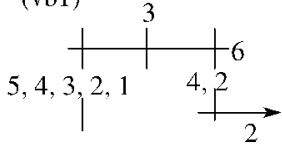

(vb5)

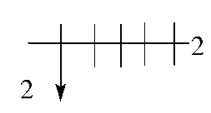

(via3)
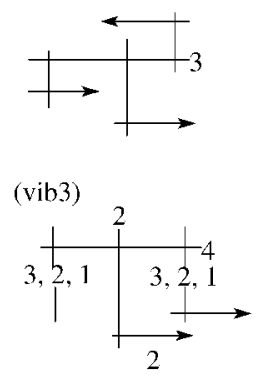

(vib4)

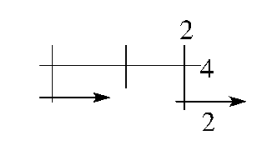

(vic2)

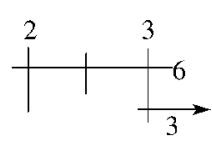

(vic3)
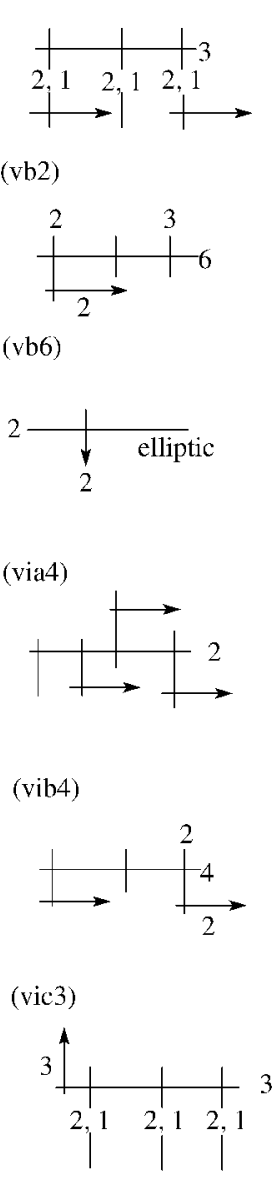

(vb2)

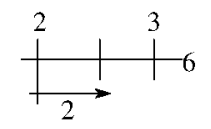

(vb6)

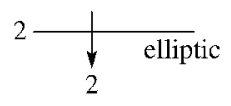

(via4)

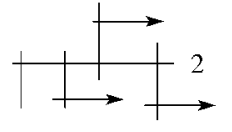

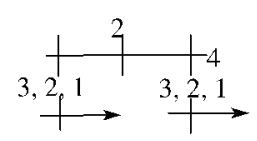

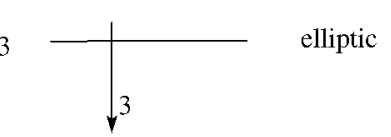


TABLE 1 (continued)

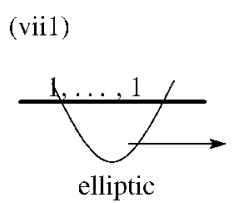

(vii5)

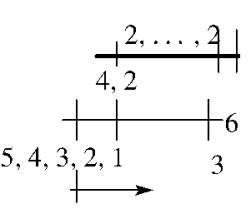

(vii2)

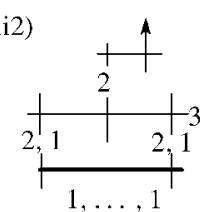

(vii6)

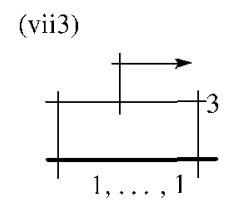

(vii7) (vii4)

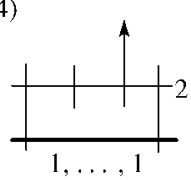

(vii8)

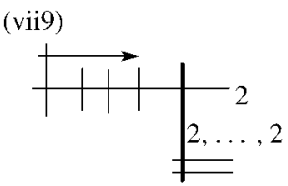

(viii1) (viii2) (viii3) (viii4)

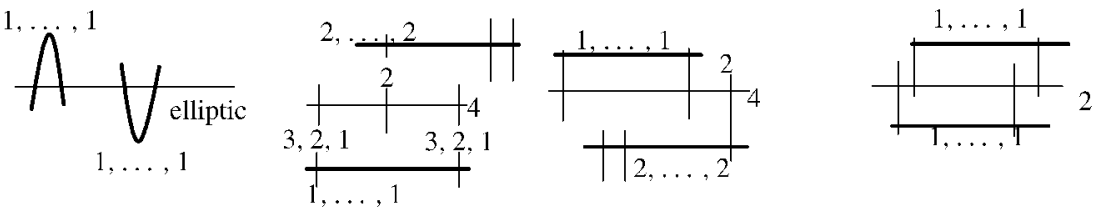

(viii5)

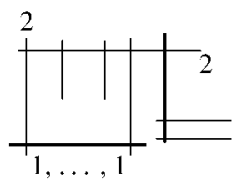

(viii8)
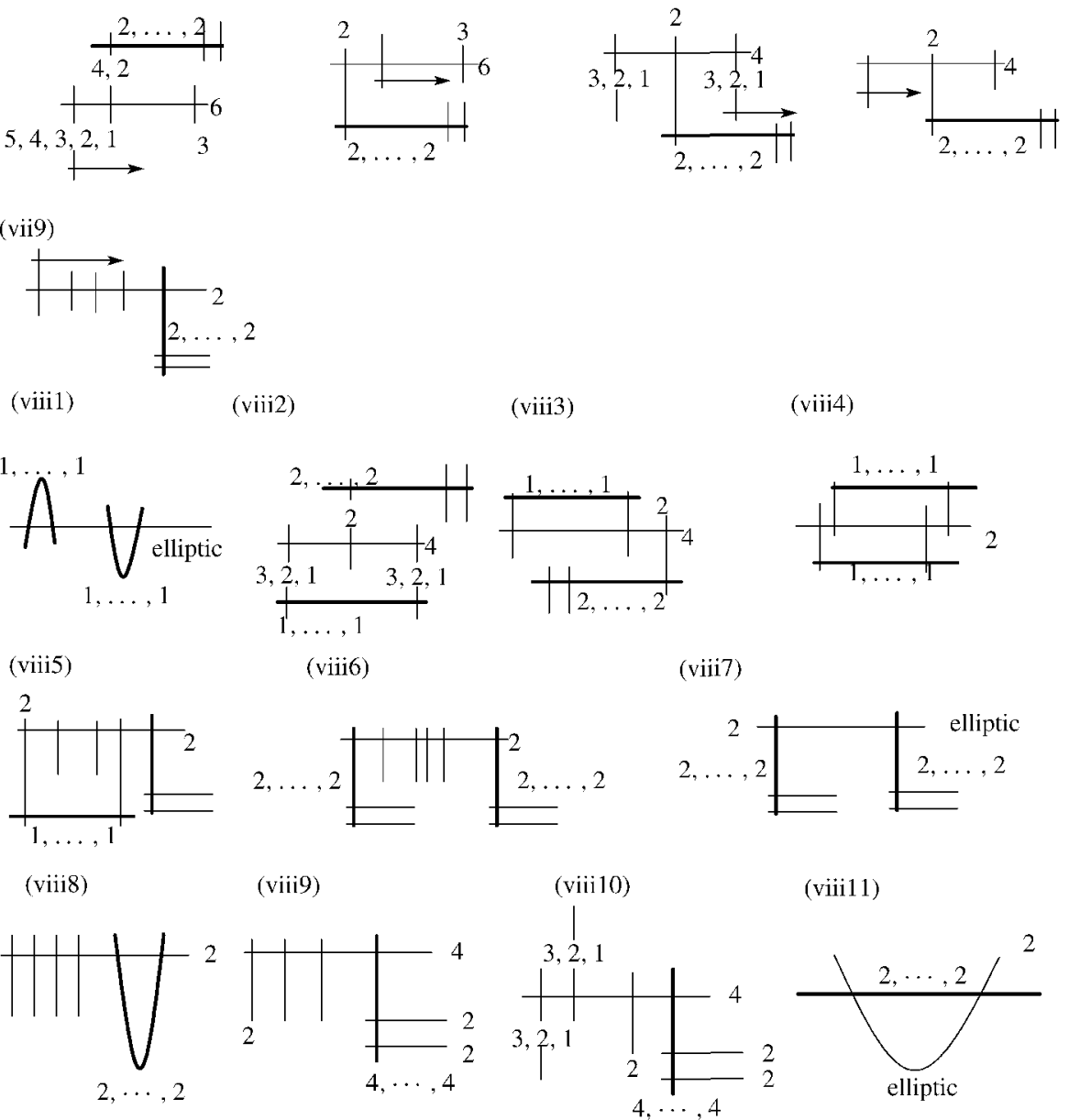

(viii12)

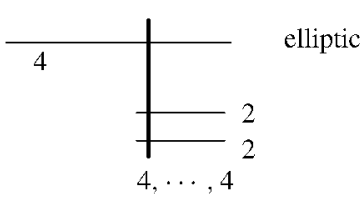


TABLE 1 (continued)
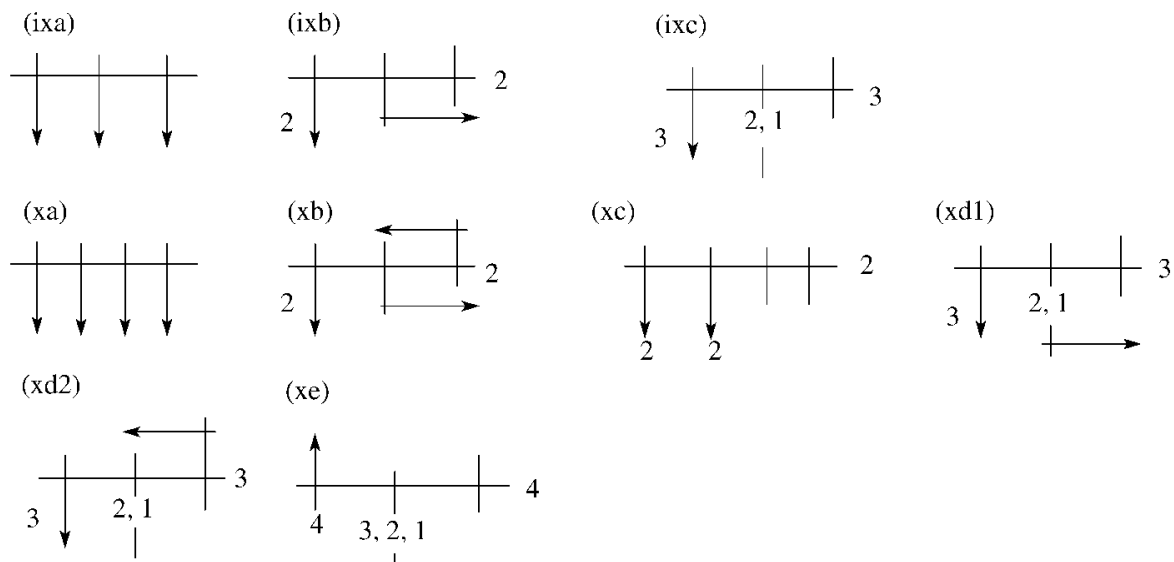

(xe)

(xi1)
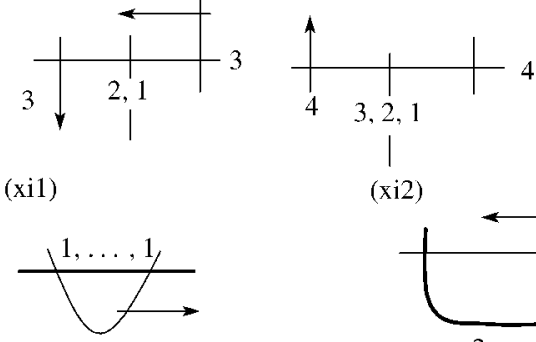

(xi2)

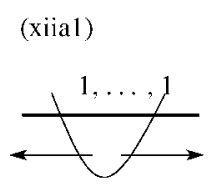

(xiiia)

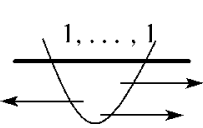

(xiv1)

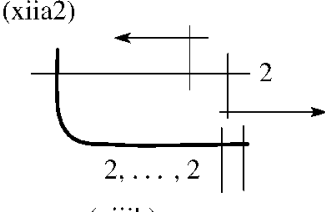

(xiib1)
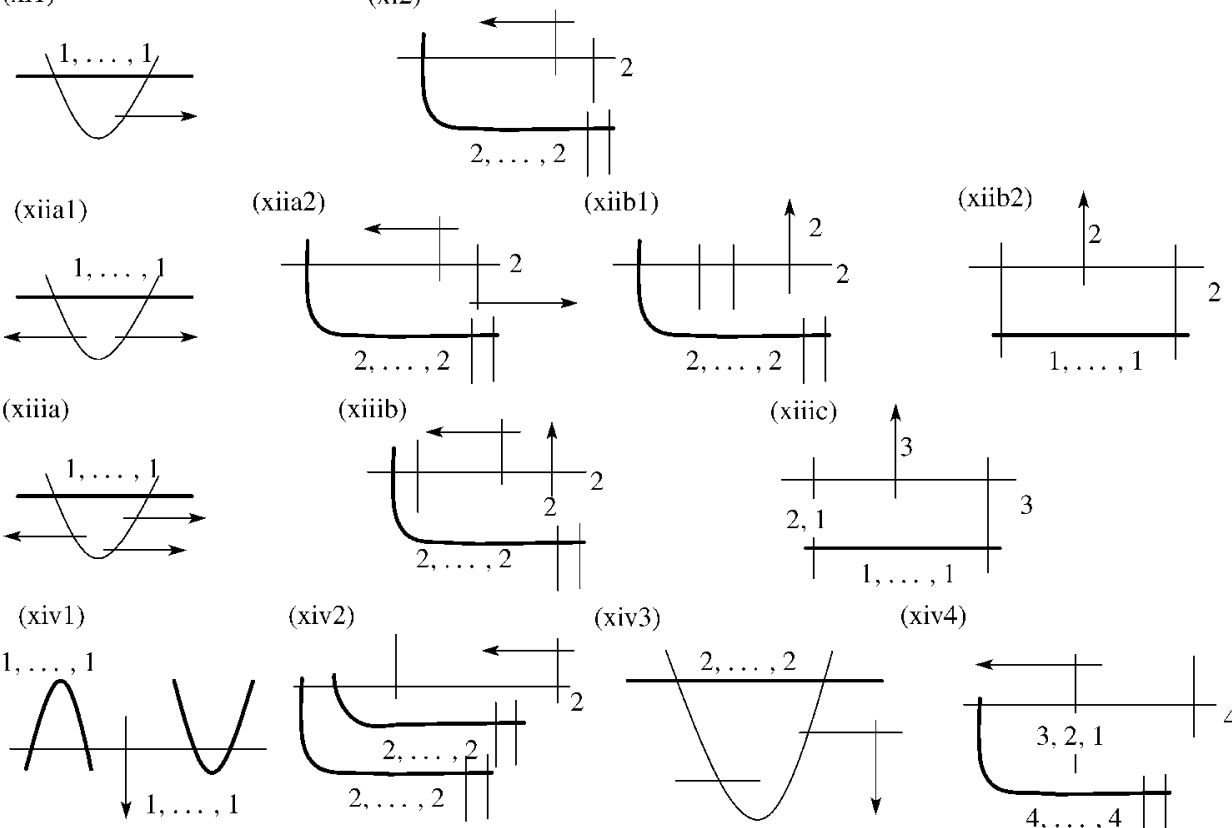

(xiv5)
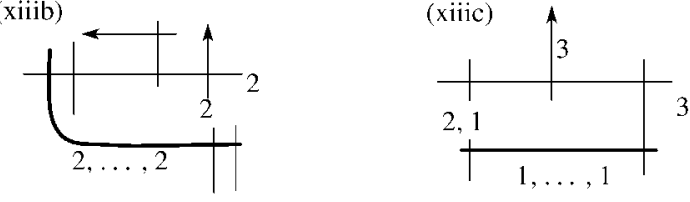

(xiv3)

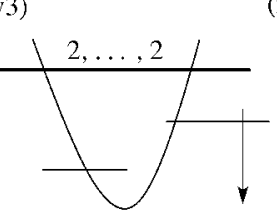

(xiv4)
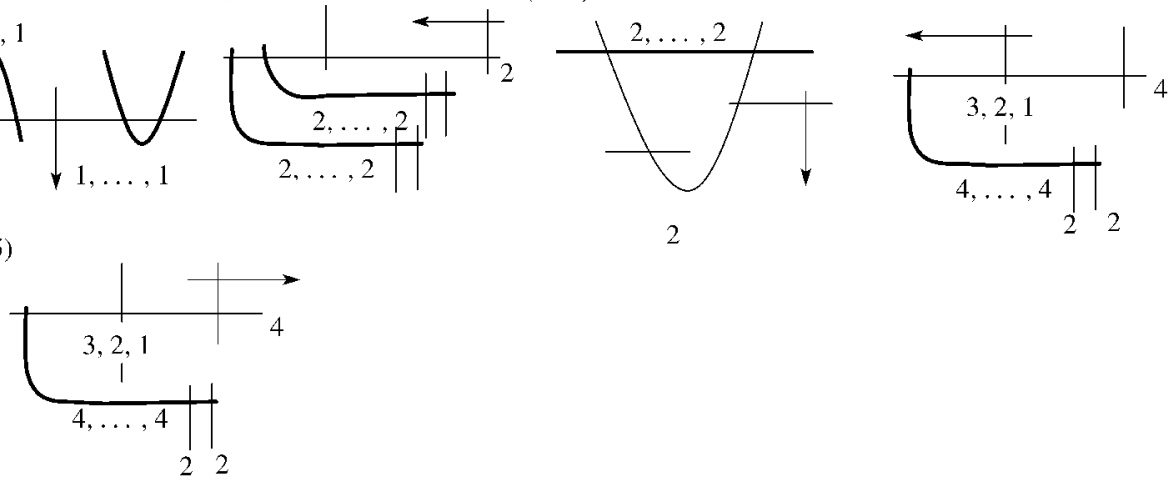
TABLE 1 (continued)

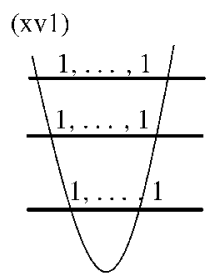

(xv5)

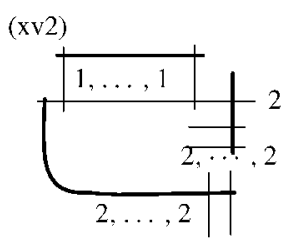

(xv6)

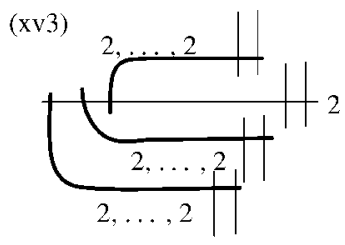

(xv4)

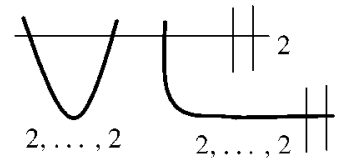

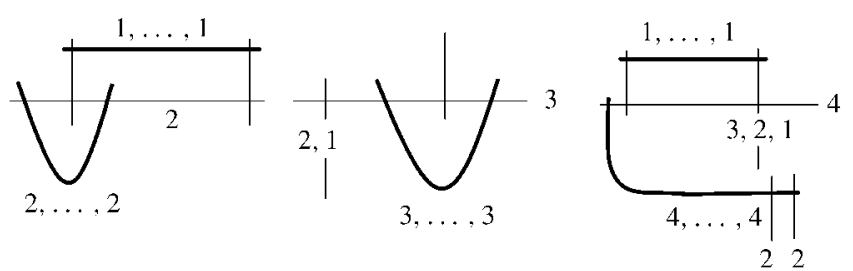

(xv8)

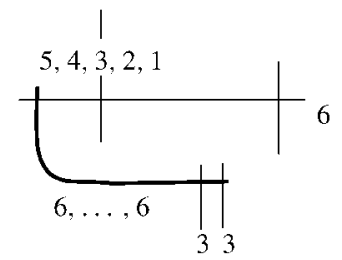

TABLE 2. Admissible system of cut curves.

(A)

(3)$$
v_{1}
$$

(B)

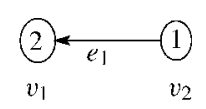

(E)

$$
\underset{v_{1}}{e_{2}} \stackrel{e_{1}}{(1)}
$$

(H)

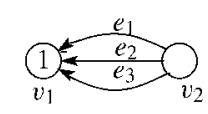

(C)

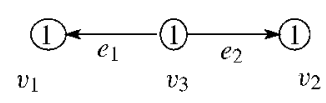

(F)

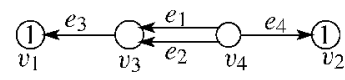

(I)

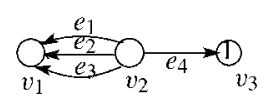

(D)

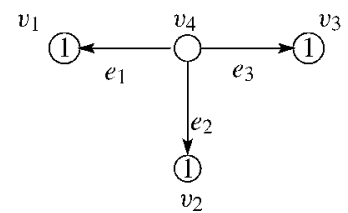

(G)

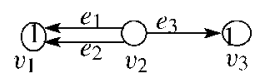

(J)

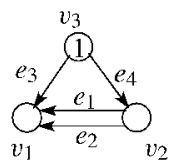


TABLE 2 (continued)

(K)

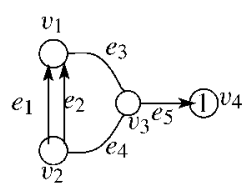

(N)

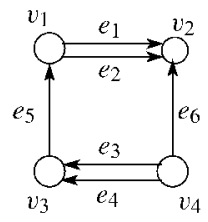

(L)

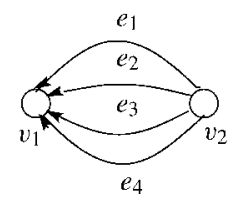

(O)

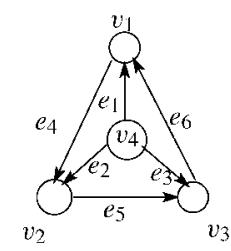

(M)

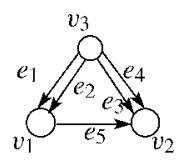

TABLE 3. Resolution of quotient graph.

(1) $\mathrm{C}_{i i j} / \mathrm{II}(1,1)$

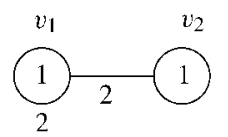

(4) $\mathrm{E}_{i j} / \mathrm{II}(0,1)$

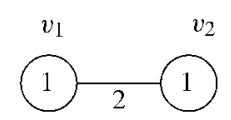

(2) $\mathrm{D}_{i i j} / \mathrm{II}(1,1)$

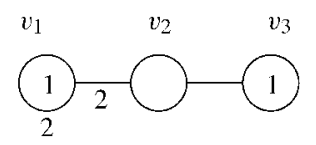

(5) $\mathrm{E}_{i i} / \mathrm{II}(1,2)$

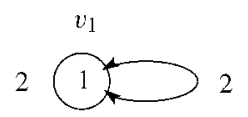

(3) $\mathrm{D}_{i i i} / \mathrm{III}(1,1)$

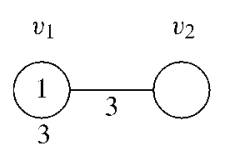

(6) $\mathrm{E}_{i i} / \Pi(1,3)$

$2 v^{v_{1}} 2<$
(7) $\mathrm{F}_{i j} / \mathrm{II}(0,1)$

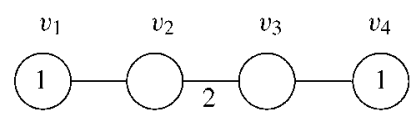

(8) $\quad \mathrm{F}_{i i} / \mathrm{II}(2,1)$

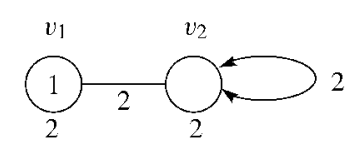


TABLE 3 (continued)
(9) $\mathrm{F}_{i i} / \mathrm{II}(2,2)$
(10) $\mathrm{G}_{i j} / \Pi(0,1)$
(11) $\mathrm{H}_{i} / \mathrm{II}(0,1)$
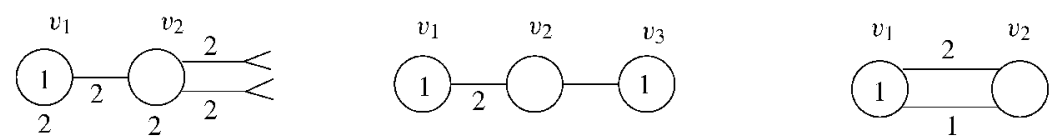

(12) $\mathrm{H}_{i} / \mathrm{III}(0,1)$

(13) $\quad \mathbf{I}_{i} / \mathrm{II}(0,1)$

(14) $\quad \mathrm{I}_{i} / \mathrm{III}(0,1)$
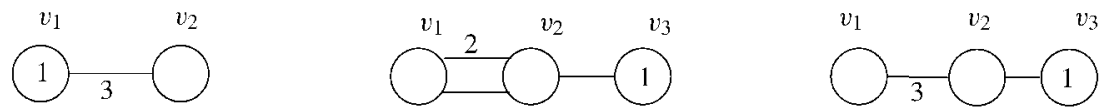

(15)

$\mathrm{J}_{i} / \mathrm{II}(0,1)$

(16) $\mathrm{J}_{i} / \mathrm{II}(1,4)$

(17) $\quad \mathrm{J}_{i} / \mathrm{II}(1,6)$
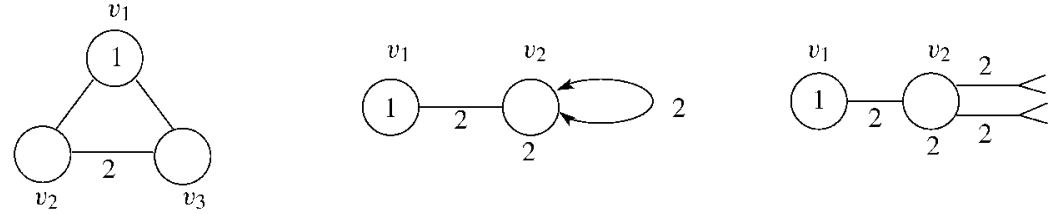

(18)

$\mathbf{K}_{i} / \mathrm{II}(0,1)$

(19) $\mathrm{K}_{i} / \mathrm{II}(1,4)$

(20) $\quad \mathrm{K}_{i} / \mathrm{II}(1,6)$
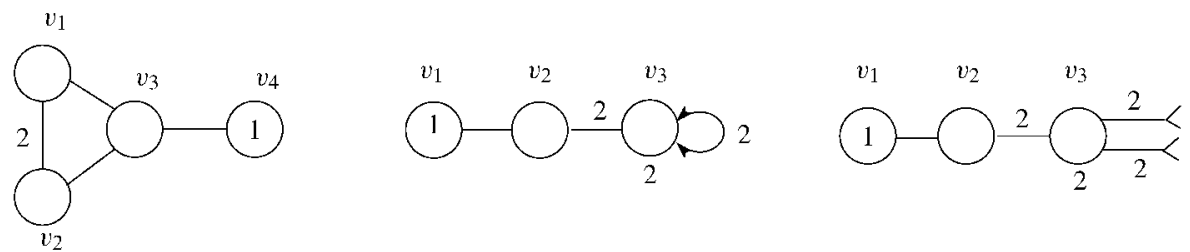

(21)

$/ / I(0,1)$

22)

$\operatorname{L/II}(0,2)$

(23) $\mathrm{L} / \mathrm{II}(1,5)$
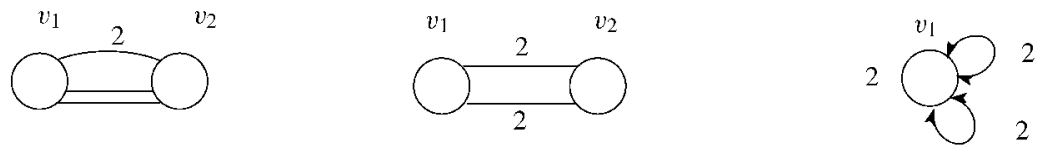

(24) $\mathrm{L} / \mathrm{II}(1,7)$

(25) $\mathrm{L} / \mathrm{II}(1,8)$

(26) $\operatorname{LIII}(0,1)$
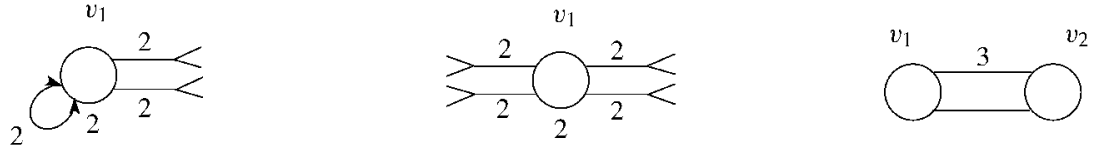
TABLE 3 (continued)

(27) $\operatorname{L} / \mathrm{IV}(0,1)$

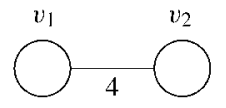

(30)

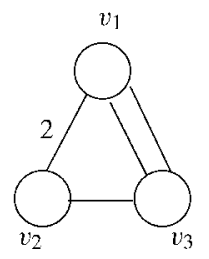

(33) $\mathrm{M} / \mathrm{IV}(1,2)$

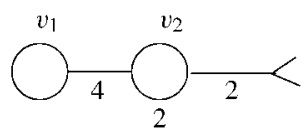

(36) $\mathrm{N} / \mathrm{IV}(0,1)$

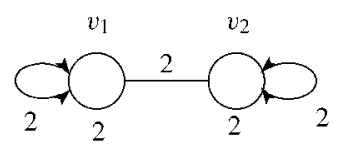

(39) $\mathrm{N} / \mathrm{II}(2,7)$

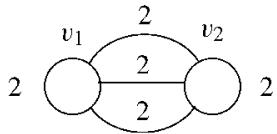

(28) $\operatorname{L} / \operatorname{IV}(1,1)$

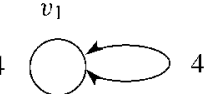

(31) $\mathrm{M} / \mathrm{II}(0,2)$

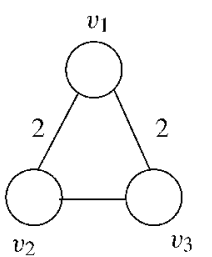

(34) $\mathrm{N} / \mathrm{II}(0,1)$

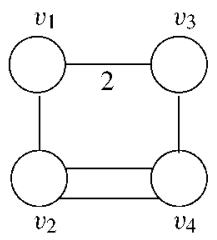

(37) $\mathrm{N} / \mathrm{II}(2,4)$

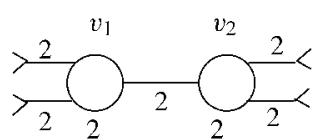

(40) $\quad \mathrm{N} / \amalg(2,8)$

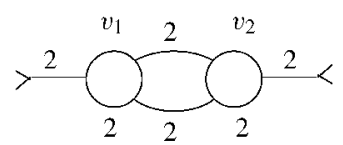

(29) $\operatorname{L} / \mathrm{VI}(1,1)$

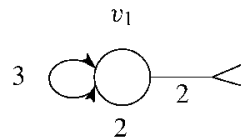

(32)

$\mathrm{M} / \mathrm{II}(1,9)$

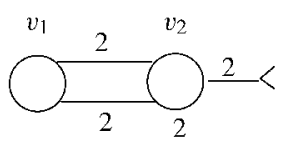

(35) $\mathrm{N} / \mathrm{II}(0,2)$

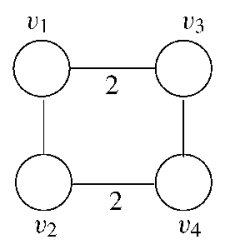

(38) $\mathrm{N} / \mathrm{II}(2,5)$

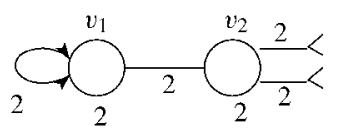

(41) $\quad \mathrm{N} / \mathrm{IV}(2,1)$

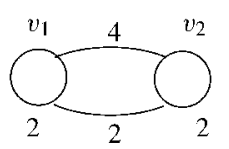


TABLE 3 (continued)

(42)

N/IV $(2,2)$

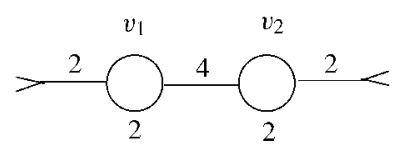

(45)

O/III $(1,2)$

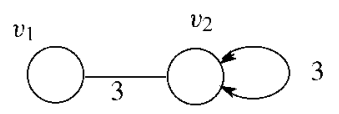

(43)

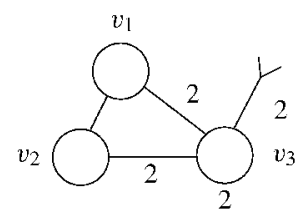

(46) $\mathrm{O} / \mathrm{IV}(1,3)$

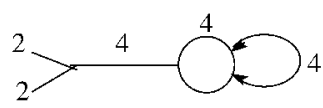

(44)

$\mathrm{O} / \mathrm{II}(2,6)$

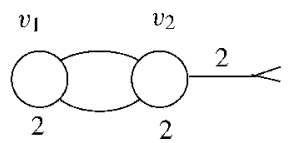

\section{REFERENCES}

[AMO] M. AsAda, M. Matsumoto AND T. OdA, Local monodromy on the fundamental groups of algebraic curves along a degenerate stable curves, J. Pure and Applied Alg. 103 (1995), 235-283.

[B1] L. BERS, Space of degenerating Riemann surfaces, Ann. of Math. Studies 79, Princeton Univ. Press, New Jersey (1975), 43-55.

[B2] L. BERS, An extremal problem for quasiconformal mappings and a theorem of Thurston, Acta Math. 141 (1978), 73-98.

[C] C. H. CLEMENS, Picard-Lefschetz theorem for families of nonsingular algebraic varieties acquiring ordinary singularities, Trans. Amer. Math. Soc. 136 (1969), 93-108.

[DM] P. Deligne AND D. MUMFord, The irreducibility of the space of curves of given genus, Publ. Math. I. H. E. S. 36 (1969), 75-110.

[ES] C. J. EARLE AND P. L. SIPE, Families of Riemann surfaces over the punctured disk, Pacific J. Math. 150 (1991), 79-86

[F] C. FABER, Chow rings of moduli spaces of curves I: The Chow ring of $\bar{M}_{3}$, Ann. of Math. 132 (1990), 331-419.

[Ha] J. HARVEY, Cyclic groups of automorphisms of compact Riemann surfaces, Quart. J. Math. Oxford Ser. (2) 17 (1966), 86-97.

[Im] Y. IMAYOSHI, Holomorphic families of Riemann surfaces and Teichmuller spaces, in Riemann surfaces and related topics, (I. Kra and B. Maskit, eds.) Ann. of Math. Studies 97 (1981), 277-300.

[Ke] S. P. KERCHHOFF, The Nielsen realization problem, Ann. of Math. 117 (1983), 235-265.

[Ko] K. KodAIRA, On compact complex analytic surfaces II, Ann. of Math. 77 (1963), 563-626.

[MM1] Y. Matsumoto And J. M. Montesinos-Amilibia, Pseudo-periodic maps and degeneration of Riemann surfaces I, II, Preprints, Univ. of Tokyo and Univ. Complutense de Madrid, 1991/1992.

[MM2] Y. Matsumoto AND J. M. Montesinos-Amilibia, Pseudo-periodic homeomorphisms and degeneration of Riemann surfaces, Bull. Amer. Math. Soc. 30 (1994), 70-75.

[Me] M. MENDES-LoPES, The relative canonical algebra for genus three fibrations, Doctoral dissertation at Univ. Warwick, 1988.

[NU1] Y. NAMIKAWA AND K. UENO, On fibers in families of curves of genus two I, singular fibers of elliptic type, in Number Theory, Algebraic Geometry and Commutative Algebra, in honor of Y. Akizuki (Y. Kusunoki, S. Mizohata, M. Nagata, H. Toda, M. Yamaguchi, H. Yoshizawa, eds.) pp. 297-371, 1973, Kinokuniya, Tokyo. 
[NU2] Y. NAmiKawa AND K. UENo, The complete classification of fibers in pencils of curves of genus two, Manuscripta Math. 9 (1973), 143-186.

[Ni1] J. NiELSEN, Die Structur periodischer Transformationen von Flächen, Mat.-Fys. Medd. Danske Vid. Selsk. 15 (1937). English translation: in Collected Papers 2, Birkhäuser, 1986.

[Ni2] J. NIELSEN, Surface transformation classes of algebraically finite type, Mat.-Fys. Medd. Danske Vid Selsk. 21 (1944), 3-89. English translation: in Collected Papers 2, Birkhäuser, 1986.

[ST] H. ShIGA AND H. TANIGAWA, On the Maskit coordinates of Teichmüller spaces and modular transformation, Kodai Math. J. 12 (1989), 437-443.

[Ta] S. TAKAMURA, Cyclic quotient construction of degenerations of complex manifolds, Preprint.

[Te] T. Terasoma, Degeneration of curves and analytic deformations, Preprint.

[Th] W. Thurston, On the geometry and dynamics of diffeomorphisms of surfaces, Bull. Amer. Math. Soc. 19 (1989), 417-431.

[Uem] K. Uematsu, Numerical classification of singular fibers in genus 3 pencils, J. Math. Kyoto Univ. 39 (1999), 763-782.

[Wim] A. WIMAN, Ueber die hyperelliptischen Kurven und diejenigen vom Geschlechte $=3$, welche eindeutige Transformationen in sich zulassen, Bihang. Kengle. Svenska Vetenkaps-Akademiens Hendlingar, Stockholm, 1895-1896.

[Win] G. B. WINTERS, On the existence of certain families of curves, Amer. J. Math. 96 (1974), 215-228.

TADASHI ASHIKAGA

DEPARTMENT OF APPLIED PHYSICS

FACULTY OF ENGINEERING

TOHOKU-GAKUIN UNIVERSITY

TAGAJO 985-8537

JAPAN

E-muil address: tashikaga@tjcc.tohoku-gakuin.ac.jp
MIZuHo IsHIZAKA

Mathematical Institute

TOHOKU UNIVERSITY

SENDAI 980-8578

JAPAN

E-mail address: 96m01@math.tohoku.ac.jp 